\title{
Tree homology and a conjecture of Levine
}

\author{
JAMES CONANT \\ ROB SCHNEIDERMAN \\ PETER TEICHNER
}

\begin{abstract}
In his study of the group of homology cylinders, J Levine [23] made the conjecture that a certain group homomorphism $\eta^{\prime}: \mathcal{T} \rightarrow \mathrm{D}^{\prime}$ is an isomorphism. Both $\mathcal{T}$ and $\mathrm{D}^{\prime}$ are defined combinatorially using trivalent trees and have strong connections to a variety of topological settings, including the mapping class group, homology cylinders, finite type invariants, Whitney tower intersection theory and the homology of $\operatorname{Out}\left(F_{n}\right)$. In this paper, we confirm Levine's conjecture by applying discrete Morse theory to certain subcomplexes of a Kontsevich-type graph complex. These are chain complexes generated by trees, and we identify particular homology groups of them with the domain $\mathcal{T}$ and range $\mathrm{D}^{\prime}$ of Levine's map.

The isomorphism $\eta^{\prime}$ is a key to classifying the structure of links up to grope and Whitney tower concordance, as explained in [6; 5]. In this paper and [3] we apply our result to confirm and improve upon Levine's conjectured relation between two filtrations of the group of homology cylinders.
\end{abstract}

57M27, 57M25; 57N10

\section{Introduction}

Let $\mathcal{T}=\mathcal{T}(m)$ be the abelian group generated by (isomorphism classes of) labeled oriented unitrivalent trees, modulo antisymmetry relations $t+(-t)=0$ and IHX relations, where $-t$ denotes the tree $t$ with the opposite orientation. Here an orientation is a cyclic ordering of the edges around each trivalent vertex and each univalent vertex is labeled by an element of the index set $\{1, \ldots, m\}$. The IHX relation happens locally around an edge connecting to trivalent vertices and is pictured in Figure 1.

A group $\mathcal{T}^{\bullet}$ is defined using the same relations, except that each generating tree also comes equipped with a labeled root, which is just a preferred univalent vertex (and is drawn as a $\bullet$ in our figures). There is an obvious homomorphism $\eta^{\prime}: \mathcal{T} \rightarrow \mathcal{T}^{\bullet}$ defined on generators by summing over all choices of roots. The group $L^{\prime}$ is defined just like $\mathcal{T}^{\bullet}$, but the root of each generator is unlabeled. There is a homomorphism 

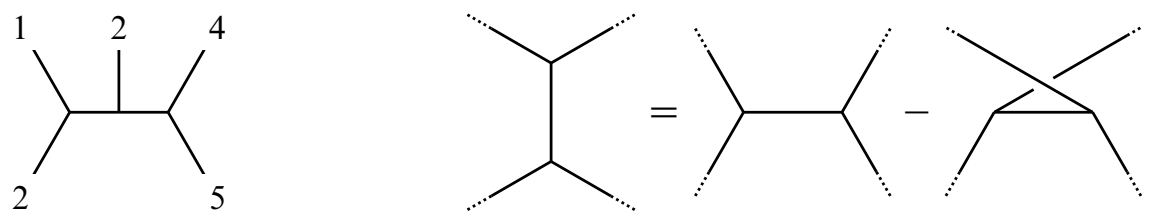

Figure 1. A tree in $\mathcal{T}(5)$, and an IHX relation

$b: \mathcal{T}^{\bullet} \rightarrow \mathrm{L}^{\prime}$ defined on generators by adding a trivalent vertex in the center of the root edge, and letting the endpoint of the new edge be the new root:

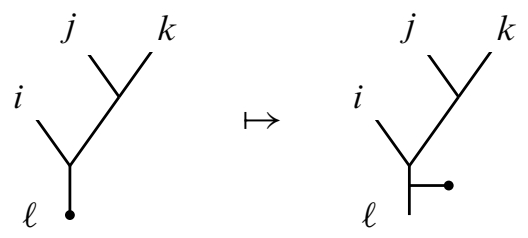

The image of $b$ consists of rooted trees with at least one trivalent vertex, and we denote this image by $L_{+}^{\prime}$. Our main result can be easily stated as follows.

Theorem 1.1 (Graphical Levine Conjecture) There is a short exact sequence

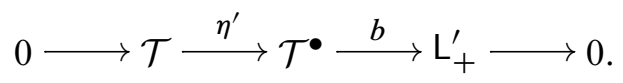

This simple-seeming statement is remarkably difficult to prove - various techniques have been tried by other authors, proving only some special cases. Levine [23] also showed the exactness in the middle of the sequence, the surjectivity of $b$ being a simple consequence of the IHX relations. Our method gives the full statement of the theorem. We interpret it in terms of combinatorially defined chain complexes called tree complexes, and apply Kozlov's [21] algebraic version of the technique of discrete Morse theory introduced by Forman [9] to these chain complexes.

The main reason why Theorem 1.1 is so useful is that it leads to a complete computation of $\mathcal{T}$, as described in Corollary 1.2 below. The structure of the analogous group with rational coefficients was previously known, and the move to integer coefficients allows access to new topological information on link concordance and homology cylinders (see eg Corollary 1.3 and Section 6).

The group $\mathcal{T}$ carries a grading by the number of trivalent vertices which we shall refer to as order. We write $\mathcal{T}_{n}$ for the order $n$ part of the grading and hence have $\mathcal{T}=\bigoplus_{n \geq 0} \mathcal{T}_{n}$. The following consequence of Theorem 1.1 will be proven in Section 1.1: 
Corollary 1.2 The groups $\mathcal{T}_{2 k}$ are free abelian (of known rank) and the torsion in $\mathcal{T}_{2 k+1}$ is generated by symmetric trees of the form $i<_{J}^{J}$ where $J$ has order $k$.

The symmetric trees above are 2-torsion by the antisymmetry relation and hence all torsion in $\mathcal{T}$ is 2 -torsion! This is one of those cherishable instances where the obvious symmetric objects actually lead to all torsion. Corollary 1.2 is proved at the end of the next Section 1.1.

Theorem 1.1 has a huge impact on our approach to understanding the graded groups $\mathrm{W}_{n}=\mathrm{W}_{n}(m)$ of the Whitney tower filtration [5] on the set of $m$-component classical links. We show that an incarnation of Cochran's [2] Bing doubling construction to realize Milnor invariants gives a realization epimorphism

$$
R_{n}: \mathcal{T}_{n} \rightarrow \mathrm{W}_{n}
$$

The main step here is to show that the algebraic relations in $\mathcal{T}$ hold in the geometric context of Whitney towers on immersed disks in the 4-ball bounded by links in the 3-sphere. Moreover, we show that the (first nonvanishing) Milnor link invariants [26; 27] induce homomorphisms

$$
\mu_{n}: \mathrm{W}_{n} \rightarrow \mathcal{T}_{n}^{\bullet}
$$

for $n$ even. The punchline is that these two homomorphisms, both involving the group $\mathrm{W}_{n}$ defined via 4-dimensional topology, compose to the purely combinatorial map $\eta_{n}^{\prime}=\mu_{n} \circ R_{n}$. The injectivity of $\eta^{\prime}$ and Corollary 1.2 hence imply directly:

Corollary 1.3 $R_{2 k}$ is an isomorphism. In particular, $\mathrm{W}_{2 k}$ is a free abelian group of known rank, classified by Milnor's invariants.

It is quite surprising that these well-known algebraic invariants detect the groups $\mathrm{W}_{2 k}$, and the main reason is that by Theorem 1.1 there is no room for further invariants.

The odd order case is more difficult and is discussed carefully in [5]. It turns out that higher-order Sato-Levine and higher-order Arf invariants are necessary to understand $W_{2 k+1}$. All of these new invariants detect some 2-torsion described in Corollary 1.2 and hence Theorem 1.1 still plays a key role in the odd order case.

\subsection{Lie algebras, quasi-Lie algebras and homology cylinders}

The graphical Levine conjecture comes from a more algebraic version and is related to a fascinating abelian group $\mathrm{D}(H)$ that lies at the heart of many areas of topology. For an abelian group $H$, define $\mathrm{D}(H)$ to be the kernel of the bracketing map $H \otimes \mathrm{L}(H) \rightarrow \mathrm{L}(H)$, where $\mathrm{L}(H)$ is the free Lie algebra generated by $H$. If $H$ carries 
a nondegenerate symplectic form, then $H \otimes \mathrm{L}(H)$ is a Lie algebra in a natural way, and $\mathrm{D}(H)$ is a Lie subalgebra. When $H=H_{1}\left(\Sigma_{g, 1} ; \mathbb{Z}\right)$ is the first homology of an oriented surface $\Sigma_{g, 1}$ of genus $g$ with one boundary component, Dennis Johnson used the Lie algebra $\mathrm{D}(H)$ to study the relative weight filtration of the mapping class group of the surface [19]. Specifically, he showed that the associated graded group of the relative weight filtration is a Lie algebra that embeds in $H \otimes \mathrm{L}(H)$, which Morita then showed (properly) embeds in $\mathrm{D}(H)$ [28]. In a different direction, letting $H$ be the direct limit of finite dimensional symplectic vector spaces, Kontsevich [20] first noticed that the homology of the Lie algebra $\mathrm{D}(H)$ can be used to study the rational homology of outer automorphism groups of free groups, a beautiful connection that was exploited by Morita [29] and then by the first author and Vogtmann [7; 8]. A nonsymplectic example, highly relevant to us, is when $H$ is the first homology of a link complement, whereupon $\mathrm{D}(H)$ becomes the natural home for Milnor invariants of the link; see our papers [5; 4] and Habiro and Massuyeau [14] and Orr [30].

Levine and Garoufalidis [11] extended Johnson's construction from the mapping class group to a group of homology cylinders, giving a diagrammatic interpretation of the Johnson homomorphisms and showing that the associated graded groups of homology cylinders become all of $\mathrm{D}(H)$ (ie the Johnson homomorphisms are epimorphisms). In related work, Habegger [13] showed that the lowest degree term of a tree-level reduction of a certain extension of the LMO invariant is given by the Johnson homomorphism. A similar result was proven by Cheptea, Habiro and Massuyeau [1] for another (functorial) extension of the LMO invariant. Levine [22] explored a different natural filtration on homology cylinders called the $Y$-filtration, originally introduced by Habiro and Goussarov [12;16] (see also Garoufalidis, Goussarov and Polyak [10]), which is defined via clasper surgery and related to finite type invariants. (See also Habiro and Massuyeau [17; 18] for more about homology cylinders.)

In order to relate the $Y$-filtration to the Johnson filtration, Levine worked with a map $\eta_{n}: \mathcal{T}_{n}(m) \rightarrow \mathrm{D}_{n}\left(\mathbb{Z}^{m}\right)=\mathrm{D}_{n}$. Rationally, $\eta_{n}$ was known to be an isomorphism by Habegger and Pitsch [15], and initially Levine thought that it was an isomorphism over the integers as well. However, in [23] he published a correction, noting that certain symmetric trees were in the kernel of $\eta_{n}$. In order to promote $\eta_{n}$ to an isomorphism, Levine realized that in the definition of $\mathrm{D}_{n}$ as the kernel of a bracketing map, one needs to replace free Lie algebras by free quasi-Lie algebras. (We note that the names of the $\eta_{n}$ and $\eta_{n}^{\prime}$ maps come from $[23 ; 24]$, and that $\eta_{n}$ was used to designate a different map in [22].)

Let $\mathrm{L}_{n}=\mathrm{L}_{n}(m)$ be the degree $n$ part of the free Lie $\mathbb{Z}$-algebra with degree 1 basis $X_{1}, \ldots, X_{m}$. It is spanned by formal nonassociative bracketing expressions of $n$ basis elements, modulo the Jacobi identity and self-annihilation relation $[Y, Y]=0$. Replacing 
this self-annihilation relation with the antisymmetry relation $[Y, Z]=-[Z, Y]$, one obtains the degree $n$ part of the free quasi-Lie algebra over $\mathbb{Z}$ on these same generators, which by the following remark, we can denote by $\mathrm{L}_{n}^{\prime}=\mathrm{L}_{n}^{\prime}(m)$. (This could also be called a quasi-Lie ring, though we follow Levine's convention in using the word "algebra.")

Remark Via the usual correspondence between labeled oriented rooted trees and nonassociative pairings given by "bracketing towards the root," we can identify the graphically defined group $\mathrm{L}^{\prime}(m)$ of Theorem 1.1 with the free quasi-Lie algebra on generators $X_{1}, \ldots, X_{m}$. Moreover, the map sending $X_{i} \otimes J$ to the rooted tree $J$ with the root labeled by $i$, gives an isomorphism of graded abelian groups

$$
\mathbb{Z}^{m} \otimes \mathrm{L}^{\prime}(m) \cong \mathrm{L}_{1}^{\prime} \otimes \mathrm{L}^{\prime}(m) \cong \mathcal{T}^{\bullet}(m)
$$

With these identifications the homomorphism $b: \mathcal{T}^{\bullet} \rightarrow \mathrm{L}^{\prime}$ of Theorem 1.1 translates into the quasi-Lie bracketing map

$$
b: \mathrm{L}_{1}^{\prime} \otimes \mathrm{L}_{n+1}^{\prime} \rightarrow \mathrm{L}_{n+2}^{\prime}, \quad X \otimes Y \mapsto[X, Y] .
$$

Let $\mathrm{D}_{n}^{\prime}=\mathrm{D}_{n}^{\prime}(m)$ be the kernel of this bracketing map. Levine [23] introduced a map $\eta_{n}^{\prime}: \mathcal{T}_{n} \rightarrow \mathrm{D}_{n}^{\prime}$ which, under the identifications in the above remark, is the same as the one we defined earlier. Levine's map $\eta^{\prime}$ is defined on generators by the formula

$$
\eta^{\prime}(t)=\sum_{v} X_{\ell(v)} \otimes B_{v}^{\prime}(t)
$$

where the sum is over all univalent vertices $v$ of $t, \ell(v)$ is the index labeling $v$, and $B_{v}^{\prime}(t)$ is defined to be the iterated bracket in $\mathrm{L}_{n+1}^{\prime}$ corresponding to the rooted oriented tree obtained from $t$ by removing the label of $v$ and letting $v$ be the root. Here is an example.

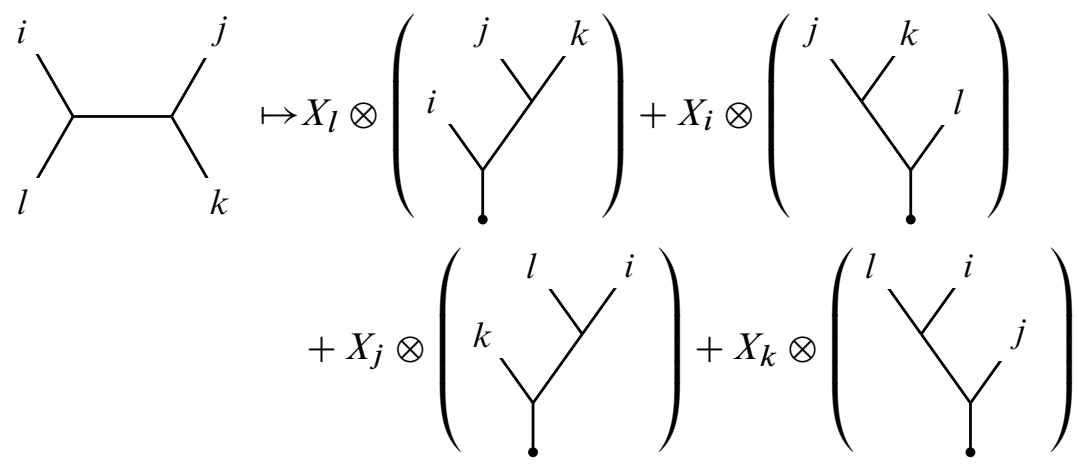


Levine showed that $\mathrm{D}_{n}^{\prime}$ is the image of $\eta_{n}^{\prime}$ and conjectured that $\eta_{n}^{\prime}: \mathcal{T}_{n} \rightarrow \mathrm{D}_{n}^{\prime}$ is an isomorphism. We are calling this the Levine Conjecture and it should now be clear that Theorem 1.1 is equivalent to this conjecture.

Theorem 1.4 (Levine Conjecture) $\eta_{n}^{\prime}: \mathcal{T}_{n} \rightarrow \mathrm{D}_{n}^{\prime}$ is an isomorphism for all $n \geq 0$.

The $n=0$ case is easy to verify, and in the proof we shall assume that $n \geq 1$. As discussed in Section 6, Theorem 1.4 allows us to prove (Theorem 6.2) and improve upon (Theorem 6.3, which we will prove in [3]) Levine's conjectured relationship between the associated graded groups of homology cylinders with respect to the Johnson filtration and the Goussarov-Habiro $Y$-filtration [23].

We remark that in Levine's notation, $\mathcal{T}_{n}=\mathcal{A}_{n}^{t}(H)$, where $H \cong \mathbb{Z}^{m}$ is the free abelian group on the index set $\{1, \ldots, m\}$, with linearity in the univalent labels. In [23], Levine obtained partial progress toward his conjecture in the form of the following theorem, and even obtained more progress in [24]. (We note that our proof of Theorem 1.4 gives an independent proof of Theorem 1.5.)

Theorem 1.5 (Levine) $\eta_{n}^{\prime}: \mathcal{T}_{n} \rightarrow \mathrm{D}_{n}^{\prime}$ is a split surjection for all $n$. Ker $\eta_{n}^{\prime}$ is the torsion subgroup of $\mathcal{T}_{n}$ if $n$ is even. It is the odd torsion subgroup if $n$ is odd. In either case

$$
(n+2) \operatorname{Ker} \eta_{n}^{\prime}=0
$$

Recall that $D_{n}$ is the kernel of the Lie algebra bracketing map $L_{1} \otimes L_{n+1} \rightarrow L_{n+2}$. According to a well-known result of Witt, $L_{n}=L_{n}(m)$ is a free abelian group of rank

$$
R_{n}:=\frac{1}{n} \sum_{d \mid n} \mu(d) \cdot m^{n / d},
$$

where $\mu(d)$ is the Möbius function; see Magnus, Karrass and Solitar [25]. Hence $\mathrm{D}_{n}=\mathrm{D}_{n}(m)$ is a free abelian group with

$$
\operatorname{rank} \mathrm{D}_{n}(m)=m \cdot R_{n+1}-R_{n+2} .
$$

By [24], $D_{2 k}^{\prime}$ is free of the same rank as $D_{2 k}$. Moreover, there is a short exact sequence

$$
0 \rightarrow \mathbb{Z}_{2} \otimes \mathrm{L}_{1} \otimes \mathrm{L}_{k+1} \rightarrow \mathrm{D}_{2 k+1}^{\prime} \rightarrow \mathrm{D}_{2 k+1} \rightarrow 0,
$$

where the left-hand map is $1 \otimes X_{i} \otimes J \mapsto X_{i} \otimes[J, J]$. This shows that $\mathrm{D}_{2 k+1}^{\prime}$ is the direct sum of the free abelian group $\mathrm{D}_{2 k+1}$ and 2-torsion generated by $X_{i} \otimes[J, J]$ for $J \in \mathrm{L}_{k+1}$. The Levine conjecture hence implies Corollary 1.2 above. 


\subsection{Outline of the proof of Theorem 1.4}

In our proof of the Levine conjecture, the first step is to reinterpret $\mathrm{L}_{n}^{\prime}$ as the zeroth homology of a chain complex $\mathbb{L}_{\bullet, n}$ of rooted labeled trees, where internal vertices may have valence higher than three, with the homological degree given by excess valence. The bracketing operation lifts to an injective map on the chain complex level, giving us a short exact sequence

$$
0 \rightarrow \mathbb{Z}^{m} \otimes \mathbb{L}_{\bullet, n+1} \rightarrow \mathbb{L}_{\bullet, n+2} \rightarrow \overline{\mathbb{L}}_{\bullet, n+2} \rightarrow 0
$$

where the complex on the right is, by definition, the cokernel. Thus we obtain the long exact sequence

$$
H_{1}\left(\mathbb{L}_{\bullet, n+2}\right) \rightarrow H_{1}\left(\overline{\mathbb{L}}_{\bullet, n+2}\right) \rightarrow \mathbb{Z}^{m} \otimes \mathrm{L}_{n+1}^{\prime} \rightarrow \mathrm{L}_{n+2}^{\prime} \rightarrow H_{0}\left(\overline{\mathbb{L}}_{\bullet, n+2}\right) \rightarrow 0 .
$$

Because the bracketing map is onto, $H_{0}\left(\overline{\mathbb{L}}_{\bullet, n+2}\right)=0$. Thus, if $H_{1}\left(\mathbb{L}_{\bullet, n+2}\right)$ were equal to zero, then we would have an alternate characterization of $\mathrm{D}_{n}^{\prime}$ as $H_{1}\left(\overline{\mathbb{L}}_{\bullet, n+2}\right)$. Indeed, in Section 4 we show that this homology does vanish:

\section{Theorem 1.6 $H_{1}\left(\mathbb{L}_{\bullet, n} ; \mathbb{Z}\right)=0$.}

With $\mathrm{D}_{n}^{\prime} \cong H_{1}\left(\overline{\mathbb{L}}_{\bullet, n+2}\right)$, the map $\eta^{\prime}$ turns into a map $\bar{\eta}: \mathcal{T}_{n} \rightarrow H_{1}\left(\overline{\mathbb{L}}_{\bullet, n+2}\right)$ which sums over adding a rooted edge to every internal vertex of a tree $t \in \mathcal{T}_{n}$.

To complete the proof of Theorem 1.4, in Section 5 we show that $H_{1}\left(\overline{\mathbb{L}}_{\bullet, n+2}\right)$ is isomorphic to $\mathcal{T}_{n}$, via a chain map $\eta_{\bullet}$. which is a lift of $\bar{\eta}$.

\section{Theorem 1.7 ๆ• induces an isomorphism $\bar{\eta}: \mathcal{T}_{n} \stackrel{\cong}{\longrightarrow} H_{1}\left(\overline{\mathbb{L}}_{\bullet, n+2}\right)$.}

The proofs of Theorem 1.6 and Theorem 1.7 use the powerful technique of discrete Morse theory for chain complexes, which we discuss in Section 3. Roughly, a discrete gradient vector field on a chain complex is a list of pairs of generators each giving combinatorial data parameterizing an atomic acyclic subcomplex. These acyclic subcomplexes can then be modded out to obtain a simpler quasi-isomorphic complex, called the Morse complex. In practice, it is often possible to find a discrete vector field that drastically reduces the size of the complex being studied. Indeed, in order to show $H_{1}\left(\mathbb{L}_{\bullet, n+2}\right)=0$, we construct a discrete vector field for which the Morse complex is 0 in degree 1 ! This vector field is inspired by the Hall basis algorithm for the free Lie algebra. Its lowest degree vectors are defined directly from this algorithm, with a suitably nice choice of a Hall order on trees. One of the conditions on a gradient vector field is that there are no "gradient loops," which in practice is often the trickiest thing to verify. In this case, the fact that the Hall basis algorithm "works" allows us to rule 
out loops involving these lowest degree vectors. The complete vector field is a natural extension of the lowest degree case, and ruling out gradient loops in general involves an exhaustive case analysis.

Actually the previous paragraph simplifies the real story somewhat, in that discrete Morse theory works well for free chain complexes, but the chain groups $\mathbb{L}_{\bullet, n+2}$ have both $\mathbb{Z}$ and $\mathbb{Z}_{2}$ direct summands. To get around this, we actually construct two slightly different vector fields, one for $\mathbb{Z}\left[\frac{1}{2}\right] \otimes \mathbb{L}_{\bullet, n+2}$ and one for $\mathbb{Z}_{2} \otimes \mathbb{L} \bullet, n+2$. The former complex kills symmetric trees of degree 0 , so that $H_{0}\left(\mathbb{L} \bullet, n+2 ; \mathbb{Z}\left[\frac{1}{2}\right]\right) \cong \mathbb{Z}\left[\frac{1}{2}\right] \otimes L_{n}$ is the free Lie algebra over $\mathbb{Z}\left[\frac{1}{2}\right]$. The second complex is more closely tied to the free quasiLie algebra, and indeed the vector field we construct for the case of $\mathbb{Z}_{2}$-coefficients is closely aligned with Levine's generalization of the Hall basis algorithm to the quasi-Lie case [23].

Now we will discuss the proof of Theorem 1.7. The first step is to generalize $\eta^{\prime}$ to a chain map

$$
\eta_{\bullet}: \mathbb{T}_{\bullet, n+2} \rightarrow \overline{\mathbb{L}}_{\bullet+1, n+2},
$$

where $\mathbb{T}_{\bullet, n+2}$ is a chain complex of unrooted trees whose zeroth homology is $\mathcal{T}_{n}$. Recall that the chain complex $\overline{\mathbb{L}}_{\bullet, n+2}$ is defined as a quotient of an abelian group of rooted trees by the image of the bracketing map. We construct a discrete vector field $\Delta$ on $\overline{\mathbb{L}}_{\bullet, n+2}$, essentially by picking a basepoint and pushing the root away from it, when possible. This gives rise to the Morse complex $\overline{\mathbb{L}}_{\bullet}^{\Delta}$, so that the composition

$$
\mathbb{T}_{\bullet, n+2} \rightarrow \overline{\mathbb{L}}_{\bullet+1, n+2} \rightarrow \overline{\mathbb{L}}_{\bullet+1, n+2}^{\Delta}
$$

has kernel and cokernel which are easy to analyze. In particular, ๆ• induces an isomorphism of homologies in degree 0 , though not in higher degrees.

The signature of a tree is an $m$-tuplet $\sigma=\left(n_{1}, \ldots, n_{m}\right)$ that records the multiplicities of each label $1, \ldots, m$. Define $|\sigma|=n_{1}+\cdots+n_{m}, \mathcal{T}_{n}=\bigoplus_{|\sigma|=n+2} \mathcal{T}_{\sigma}$, where $\mathcal{T}_{\sigma}$ is the subgroup of $\mathcal{T}_{n}$ spanned by trees with signature $\sigma$. For the reader's amusement, in Figure 2, we list computer calculations of the $\mathcal{T}_{\sigma}$ groups for small values of $\sigma=(j, k)$.

Acknowledgements This paper was written while the first two authors were visiting the third author at the Max-Planck-Institut für Mathematik in Bonn. They all thank MPIM for its stimulating research environment and generous support. The first author was partially supported by NSF grant DMS-0604351 and the last author was also supported by NSF grants DMS-0806052 and DMS-0757312. We thank Daniel Moskovich and the referee for their detailed comments. 


\begin{tabular}{l|llllllll} 
& 2 & 3 & 4 & 5 & 6 & 7 & 8 & 9 \\
\hline 2 & $\mathbb{Z}$ & $\mathbb{Z}_{2}$ & $\mathbb{Z}$ & $\mathbb{Z}_{2}$ & $\mathbb{Z}$ & $\mathbb{Z}_{2}$ & $\mathbb{Z}$ & $\mathbb{Z}_{2}$ \\
3 & $\mathbb{Z}_{2}$ & $\mathbb{Z}$ & $\mathbb{Z}_{2}$ & $\mathbb{Z}$ & $\mathbb{Z} \oplus \mathbb{Z}_{2}$ & $\mathbb{Z}$ & $\mathbb{Z} \oplus \mathbb{Z}_{2}$ & $\mathbb{Z}^{2}$ \\
4 & $\mathbb{Z}$ & $\mathbb{Z}_{2}$ & $\mathbb{Z}^{2}$ & $\mathbb{Z} \oplus \mathbb{Z}_{2}$ & $\mathbb{Z}^{3}$ & $\mathbb{Z}^{2} \oplus \mathbb{Z}_{2}^{2}$ & $\mathbb{Z}^{5}$ & $\mathbb{Z}^{3} \oplus \mathbb{Z}_{2}^{2}$ \\
5 & $\mathbb{Z}_{2}$ & $\mathbb{Z}$ & $\mathbb{Z} \oplus \mathbb{Z}_{2}$ & $\mathbb{Z}^{3}$ & $\mathbb{Z}^{3} \oplus \mathbb{Z}_{2}^{2}$ & $\mathbb{Z}^{6}$ & $\mathbb{Z}^{7} \oplus \mathbb{Z}_{2}^{2}$ & $\mathbb{Z}^{11}$ \\
6 & $\mathbb{Z}$ & $\mathbb{Z} \oplus \mathbb{Z}_{2}$ & $\mathbb{Z}^{3}$ & $\mathbb{Z}^{3} \oplus \mathbb{Z}_{2}^{2}$ & $\mathbb{Z}^{9}$ & $\mathbb{Z}^{9} \oplus \mathbb{Z}_{2}^{3}$ & $\mathbb{Z}^{19}$ & $\mathbb{Z}^{22} \oplus \mathbb{Z}_{2}^{5}$ \\
7 & $\mathbb{Z}_{2}$ & $\mathbb{Z}$ & $\mathbb{Z}^{2} \oplus \mathbb{Z}_{2}^{2}$ & $\mathbb{Z}^{6}$ & $\mathbb{Z}^{9} \oplus \mathbb{Z}_{2}^{3}$ & $\mathbb{Z}^{19}$ & $\mathbb{Z}^{28} \oplus \mathbb{Z}_{2}^{5}$ & $\mathbb{Z}^{47}$ \\
8 & $\mathbb{Z}$ & $\mathbb{Z} \oplus \mathbb{Z}_{2}$ & $\mathbb{Z}^{5}$ & $\mathbb{Z}^{7} \oplus \mathbb{Z}_{2}^{2}$ & $\mathbb{Z}^{19}$ & $\mathbb{Z}^{28} \oplus \mathbb{Z}_{2}^{5}$ & $\mathbb{Z}^{58}$ & \\
9 & $\mathbb{Z} 2$ & $\mathbb{Z}^{2}$ & $\mathbb{Z}^{3} \oplus \mathbb{Z}_{2}^{2}$ & $\mathbb{Z}^{11}$ & $\mathbb{Z}^{22} \oplus \mathbb{Z}_{2}^{5}$ & $\mathbb{Z}^{47}$ & & \\
10 & $\mathbb{Z}$ & $\mathbb{Z} \oplus \mathbb{Z}_{2}$ & $\mathbb{Z}^{7}$ & $\mathbb{Z}^{13} \oplus \mathbb{Z}_{2}^{3}$ & $\mathbb{Z}^{36}$ & & & \\
11 & $\mathbb{Z} 2$ & $\mathbb{Z}^{2}$ & $\mathbb{Z}^{5} \oplus \mathbb{Z}_{2}^{3}$ & $\mathbb{Z}^{18}$ & & & & \\
12 & $\mathbb{Z}$ & $\mathbb{Z}^{2} \oplus \mathbb{Z}_{2}$ & $\mathbb{Z}^{9}$ & & & & &
\end{tabular}

Figure 2. A computer generated table of the groups $\mathcal{T}_{(j, k)}$

\section{Tree homology}

In this section, we interpret Levine's conjecture in a homological setting. It is wellknown that the free (quasi)-Lie algebra can be regarded as the zeroth homology of a complex of rooted oriented trees (of arbitrary valence $\neq 2$ ), with univalent vertices labeled by the generators, since the boundary of a tree with a 4-valent vertex is precisely a Jacobi relator. Over the rationals, all the homology is concentrated in degree zero (Proposition 2.6), but the integral homology appears to be unknown.

Definition 2.1 Throughout this paper, trees are allowed to have vertices of any valence other than 2, and are considered up to isomorphism.

An orientation of a tree is an equivalence class of orderings of the edges, where two orderings are equivalent if they differ by an even permutation. Each tree has at most two orientations, and one is said to be the negative of the other. See the remark below for discussion of why this is equivalent to the usual notion.

Using the previous notation for unitrivalent trees, labels from the index set $\{1,2, \ldots, m\}$ are used to decorate univalent vertices, and a rooted tree has all univalent vertices labeled except for a single unlabeled root univalent vertex. All nonroot univalent vertices are called leaves, and all vertices of valence $\geq 3$ all called internal vertices.

The bracket of two oriented rooted trees is the rooted tree $\left(J_{1}, J_{2}\right)$ defined by identifying the roots of $J_{1}$ and $J_{2}$ and attaching an edge to the identified vertex, the other vertex of this edge being the new root. The orientation is given by ordering the root edge first, 
then the edges of $J_{1}$ in the order prescribed by $J_{1}$ 's orientation, and finally the edges of $J_{2}$ in the order prescribed by its orientation.

The homological degree of a tree is defined to be $\sum_{v}(|v|-3)$ where the sum is over all internal vertices $v$, and $|v|$ represents the valence of the vertex.

Remark Recall, that for finite-dimensional vector spaces $V, \operatorname{det}(V):=\bigwedge^{\operatorname{dim}(V)} V$, and that an orientation for $V$ is a choice of basis element of $\operatorname{det}(V)$ up to multiplication by positive scalars. So, we can think of an orientation of a tree as an orientation of the vector space $\mathbb{R}^{\mathcal{E}}$, where $\mathcal{E}$ is the set of edges of the tree, or more specifically as a choice of basis element for $\operatorname{det}\left(\mathbb{R}^{\mathcal{E}}\right)$. A more standard orientation for unitrivalent trees is given by ordering the half-edges around a vertex. More precisely, if we let $H(v)$ denote the set of half-edges incident to $v$, and $\mathcal{V}$ be the set of vertices of the tree, it is an orientation of the vector space $\bigotimes_{v \in \mathcal{V}} \mathbb{R}^{H(v)}$. According to Conant and Vogtmann [7, Proposition 2], these are equivalent notions for odd-valent trees, but for the reader's convenience we give an argument below in Proposition 2.2 as well.

Proposition 2.2 The two notions of orientation described above coincide for unitrivalent trees. Indeed $\operatorname{det}\left(\mathbb{R}^{\mathcal{E}}\right)$ is canonically isomorphic to $\operatorname{det}\left(\bigotimes_{v \in \mathcal{V}} \mathbb{R}^{H(v)}\right)$.

Proof In the following proof, we refer to our notion of orientation as an "edgeordering," and to the other one as a "vertex-orientation." First we recursively define a special type of edge-ordering which will be compatible with a vertex-orientation. For trees with a single edge, there is only one edge-ordering and one vertex-orientation, which we declare to be compatible. In the general case, let $J$ be a tree, and fix a univalent vertex as the root. Looking at the trivalent vertex, $v$, next to the root, there are three trees emanating from $v$, the root edge, followed by $J_{1}$, followed by $J_{2}$. Let the roots of $J_{1}$ and $J_{2}$ be $v$. Then order the edges of $J$ by numbering the root edge first, followed by the edges of $J_{1}$ in the recursively specified order, followed by the edges of $J_{2}$ in the recursively specified order. Because a unitrivalent tree has an odd number of edges, changing the vertex-orientation at a single vertex will also flip the edge-ordering.

With this preliminary construction under our belt, we say that an edge-ordering is compatible with a vertex-orientation if it is of the above form for some choice of root, or if there is a trivalent vertex $v$ with emanating trees, in (cyclic) order, $J_{1}, J_{2}, J_{3}$, such that the edge-ordering restricted to each subtree is of the above form with root $v$, and the edges of $J_{1}$ lie before those of $J_{2}$ which lie before those of $J_{3}$. We claim that any two edge-orderings compatible with a given vertex-orientation are equivalent. We do this by checking that changing $J_{1}, J_{2}, J_{3}$ by a cyclic permutation gives an 
equivalent edge-orientation, and that switching the base vertex $v$ to an adjacent vertex also gives an equivalent edge-ordering. Changing the order by the cyclic permutation $J_{1}, J_{2}, J_{3} \mapsto J_{2}, J_{3}, J_{1}$ gives an equivalent edge-ordering: if $e_{i}$ denotes the number of edges of $J_{i}$, the sign is given by $(-1)^{e_{1}\left(e_{2}+e_{3}\right)}$, which is +1 because each tree has an odd number of edges. Now suppose we want to switch the base vertex from one trivalent vertex to another, adjacent one. This is depicted in the following picture where the cyclic orientation is induced by the clockwise orientation of the plane. The bold vertex is the chosen base vertex in each case, and the trees are numbered so that a lower index indicates that the edges of one lie before the edges of another. So for example, as trees $J_{3}=J_{1}^{\prime}$, but the numbering of the edges is different. The central edge is numbered first in the edge-ordering, indicated by the label 1 in the center.
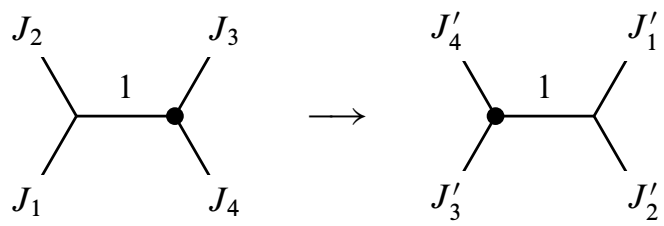

Thus, the edge-order differs by the even permutation $\left(J_{1}, J_{2}, J_{3}, J_{4}\right) \mapsto\left(J_{3}^{\prime}, J_{4}^{\prime}, J_{1}^{\prime}, J_{2}^{\prime}\right)$ which is an equivalence. On the other hand, if we want to move the base vertex from a trivalent one to a univalent one, the edge-order remains unchanged.

In what follows, rooted trees will usually be denoted by capital letters, and unrooted trees by lower case letters.

Definition 2.3 In the following chain complexes, we divide by the relation $(T,-$ or $)=$ $-(T$, or) for every oriented tree $(T$, or $)$.

For all $k \geq 2$, let $\mathbb{L}_{\bullet, k}$ be the chain complex spanned by oriented rooted trees with $k+1$ total univalent vertices, and the $k$ leaves labeled by the cardinality $m$ index set. The trees are graded by homological degree.

Let $v$ be an internal vertex of a tree $J$ of valence $\geq 4$, and let $P$ be an unordered partition of the half-edges incident to $v$ into two sets each having at least two elements. The partition $P$ determines an expansion of $J$, where the vertex $v$ expands into a new edge $e$, and the half-edges are distributed to the ends of $e$ according to the partition $P$. The induced orientation of an expansion is defined by numbering the new edge first, and increasing the numbering of the other edges by one.

The boundary operator $\partial: \mathbb{L}_{\bullet, k} \rightarrow \mathbb{L}_{\bullet-1, k}$ is defined by setting $\partial J$ equal to the sum of all expansions of $J$. See Figure 3, which shows the three expansions of a 4-valent vertex. The fact that $\partial^{2}=0$ comes from the fact that terms of $\partial^{2}(J)$ consist of 
expanding two edges. These terms come in pairs, based on which edge is expanded first, and have opposite orientation, so cancel. Note that $\partial$ vanishes on degree zero trees since they have only trivalent internal vertices.

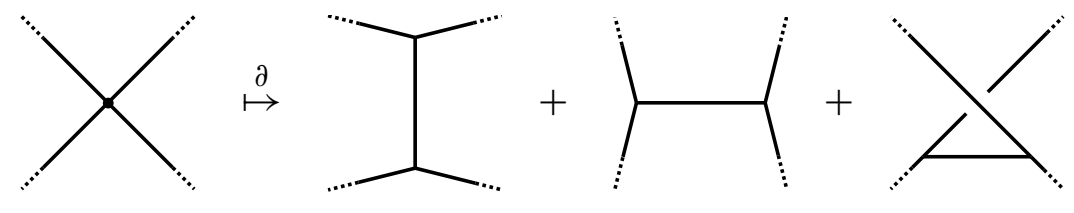

Figure 3. An IHX relation appearing as the image of $\partial$

The chain complex $\mathbb{T}_{\bullet, k}$ is spanned by oriented labeled unrooted trees with $k$ leaves. The trees are again graded by homological degree. The boundary operator $\partial: \mathbb{T}_{\bullet, k} \rightarrow$ $\mathbb{T}_{\bullet-1, k}$ is defined as before by setting $\partial t$ equal to the sum of all expansions of $t$.

The chain complex $\overline{\mathbb{L}}_{\bullet, k}$ is the quotient complex of $\mathbb{L}_{\bullet, k}$ by the subcomplex spanned by trees of the form $(i, J)=-<_{J}^{i}$. Here the notation $i$ stands for the rooted tree having a single $i$-labeled leaf.

Some of the homology groups of these chain complexes turn out to be relevant to us.

Proposition 2.4 We have the following isomorphisms ( $\mathbb{Z}$-coefficients):

(1) $H_{0}\left(\mathbb{L}_{\bullet, n}\right) \cong \mathrm{L}_{n}^{\prime}$

(2) $H_{0}\left(\mathbb{T}_{\bullet, n+2}\right) \cong \mathcal{T}_{n}$

(3) $H_{1}\left(\overline{\mathbb{L}}_{\bullet, n+2}\right) \cong \mathrm{D}_{n}^{\prime}$ for $n>0$.

Proof The first isomorphism comes from the fact that in homological degree 0 all trees are trivalent, and hence they are all cycles. The image of the boundary operator is precisely the submodule of IHX relators, since $\partial$ expands a 4-valent vertex into an IHX relator (Figure 3). Although the signs are verified in [7, page 1207], we give an argument here based on the concrete construction of Proposition 2.2. Suppose the trees emanating from the given $4-$ valent vertex in Figure 3 are $J_{1}, J_{2}, J_{3}, J_{4}$, starting in the top left and proceeding clockwise. Suppose the tree has an edge-ordering which respects the order indicated by the indices. Now after expanding the vertex, the central edge is numbered 1 . The first tree in the expansion has a compatible edge order (where the bottom vertex is the base) with the clockwise orientation on each trivalent vertex. To make the second tree have a compatible edge order (with the right-hand vertex as the base) we need to make the reordering $\left(J_{1}, J_{2}, J_{3}, J_{4}\right) \mapsto\left(J_{2}^{\prime}, J_{3}^{\prime}, J_{4}^{\prime}, J_{1}^{\prime}\right)$, which is an odd permutation, yielding a minus sign. Finally, to get a compatible edge order in the third tree with the right-hand vertex as the base, we need to make the even reordering 
$\left(J_{1}, J_{2}, J_{3}, J_{4}\right) \mapsto\left(J_{3}^{\prime}, J_{2}^{\prime}, J_{4}^{\prime}, J_{1}^{\prime}\right)$. So we recover the usual signs in the IHX relation and Jacobi identity: $I-H+X=0$.

The second isomorphism is similar.

We proceed to explain the third isomorphism, which depends on Theorem 1.6. Recall that by definition $\mathrm{D}_{n}^{\prime}$ is the kernel of the bracketing map

$$
\mathbb{Z}^{m} \otimes \mathrm{L}_{n+1}^{\prime} \rightarrow \mathrm{L}_{n+2}^{\prime}
$$

via the identification $L_{1}^{\prime} \cong \mathbb{Z}^{m}$. This bracketing operation on the quasi-Lie algebra lifts to a chain map

$$
\mathbb{B r}_{\bullet}: \mathbb{Z}^{m} \otimes \mathbb{L}_{\bullet, n+1} \rightarrow \mathbb{L}_{\bullet, n+2}
$$

which sends $X_{i} \otimes J$ to the oriented tree $(i, J)$. Note that for $n>0, \mathbb{B r} r_{\bullet}$ is injective at the chain level. Thus we get a short exact sequence of chain complexes

$$
0 \rightarrow \mathbb{Z}^{m} \otimes \mathbb{L}_{\bullet, n+1} \rightarrow \mathbb{L}_{\bullet, n+2} \rightarrow \overline{\mathbb{L}}_{\bullet, n+2} \rightarrow 0,
$$

where $\overline{\mathbb{L}}_{\bullet, n+2}$ is, by definition, the cokernel of $\mathbb{B} r_{\bullet}$. Using statement (1) of the proposition, this gives rise to the long exact sequence

$$
H_{1}\left(\mathbb{L}_{\bullet, n+2}\right) \rightarrow H_{1}\left(\overline{\mathbb{L}}_{\bullet, n+2}\right) \rightarrow \mathbb{Z}^{m} \otimes \mathrm{L}_{n+1}^{\prime} \rightarrow \mathrm{L}_{n+2}^{\prime} \rightarrow H_{0}\left(\overline{\mathbb{L}}_{\bullet, n+2}\right) .
$$

We will prove later (Theorem 1.6) that $H_{1}\left(\mathbb{L}_{\bullet, n+2}\right)=0$. Since the bracketing map is onto, we get the short exact sequence

$$
0 \rightarrow H_{1}\left(\overline{\mathbb{L}}_{\bullet, n+2}\right) \stackrel{\kappa}{\rightarrow} \mathbb{Z}^{m} \otimes \mathrm{L}_{n+1}^{\prime} \rightarrow \mathrm{L}_{n+2}^{\prime} \rightarrow 0,
$$

where $\kappa$ is the connecting homomorphism from the long exact sequence. Hence $\mathrm{D}_{n}^{\prime} \cong H_{1}\left(\overline{\mathbb{L}}_{\bullet, n+2}\right)$.

Let us interpret $\eta_{n}^{\prime}$ in this context. Clearly $\eta_{n}^{\prime}$ lifts uniquely to a map $\bar{\eta}_{n}$ as in the diagram below:

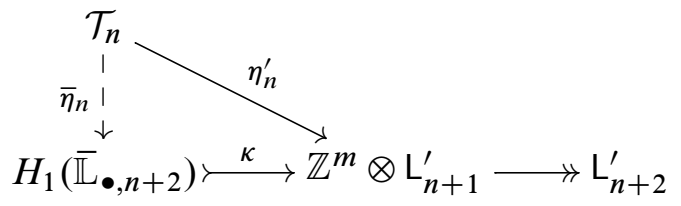

Suppose $t \in \mathbb{T}_{0, n+2}$ is an oriented tree. Define $t^{r} \in \mathbb{L}_{1, n+2}$ to be the sum of adding a root edge, numbered first in the orientation, to all of the internal vertices of $t$ :

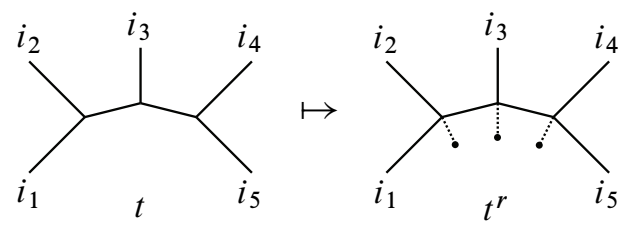


The dotted edges mean that we are summing over putting the root edge in each position. We claim that $\bar{\eta}_{n}(t)=t^{r}$. So we must verify that $\kappa\left(t^{r}\right)=\eta_{n}^{\prime}(t)$. The map $\kappa$ is defined via the snake lemma as in the diagram below.

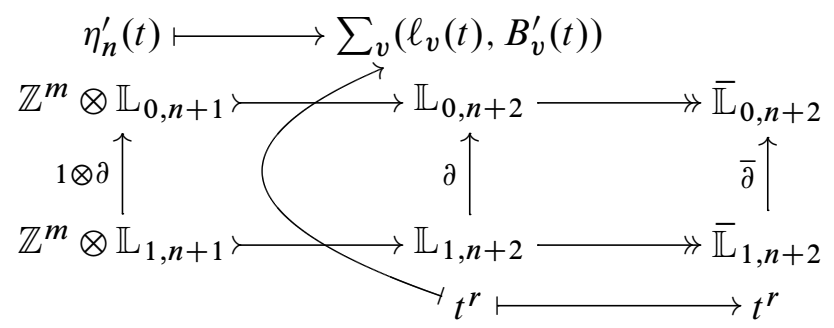

Here we use that $\partial t^{r}=\sum_{v}\left(\ell_{v}(t), B_{v}^{\prime}(t)\right)$ because of internal cancellation of the root:

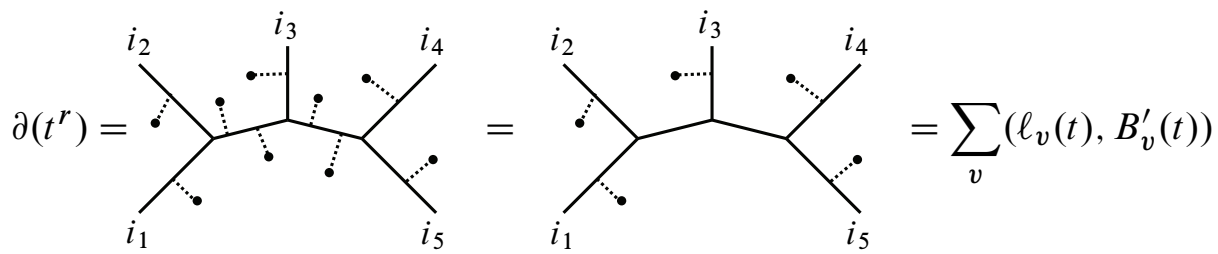

To see the cancellation, note that there are two ways to add a rooted edge to an internal edge, depending on which of the two endpoints it is being expanded from. These two trees have opposite orientation: if the edge was originally numbered $i$, then the numbering of the bisected edge will be $(1, i+1)$ in one term and $(i+1,1)$ in the other, which differ by a transposition, so have opposite sign. Next we check that the signs of the resulting sum $\sum_{v}\left(\ell_{v}(t), B_{v}^{\prime}(t)\right)$ are correct. Below is depicted the result of expanding the root edge out toward a univalent vertex $v$, along an edge which is numbered $j-1$.

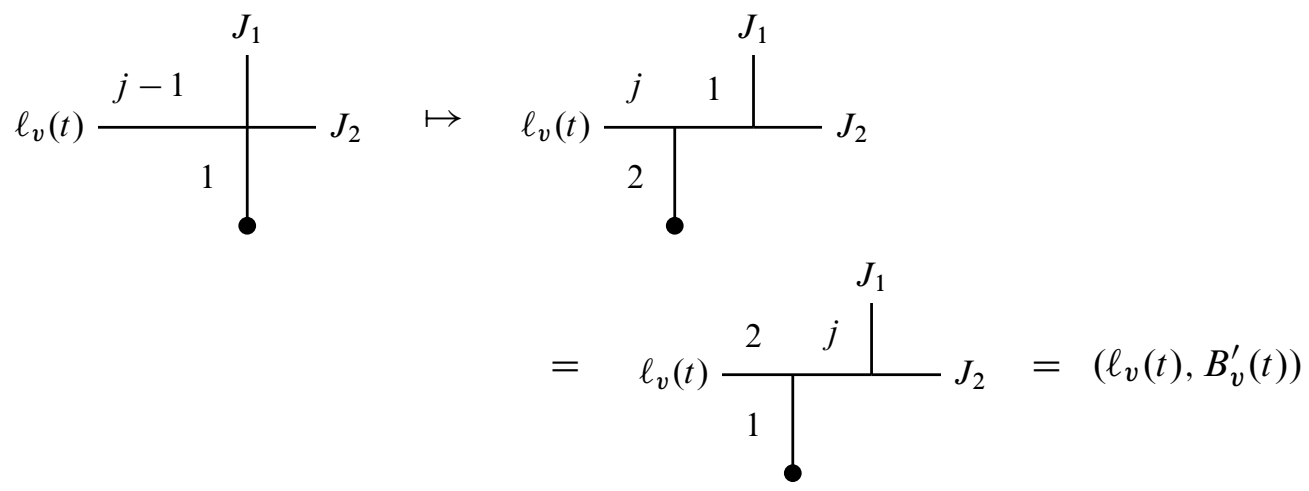

Thus the sign is indeed +1 as claimed. 
Now tracing through the diagram shows that $t^{r} \mapsto \eta_{n}^{\prime}(t)$.

In fact, $\bar{\eta}$ extends to a map of chain complexes, a fact which we will need in Section 5. Define $\eta_{\bullet}: \mathbb{T}_{\bullet, n} \rightarrow \overline{\mathbb{L}}_{\bullet+1, n}$ by letting $\eta_{\bullet}(t)$ be the sum over attaching a root edge to every internal vertex of $t$, multiplied by the sign $(-1)^{\operatorname{deg}(t)}$.

\section{Lemma $2.5 \eta_{\bullet}: \mathbb{T}_{\bullet, n} \rightarrow \overline{\mathbb{L}}_{\bullet+1, n}$ is a chain map.}

Proof For any internal vertex $v$ of an unrooted tree $t$, let $\alpha_{v}(t)$ denote the rooted tree gotten by attaching a root edge to $v$, so that $\eta_{\bullet}(t)=(-1)^{\operatorname{deg}(t)} \sum_{v} \alpha_{v}(t)$. By definition $\partial \eta_{\bullet}(t)$ is a sum of expansions of $\eta_{\bullet}(t)$, and since those expansions which push the root edge onto an interior edge of $t$ all cancel in pairs as in the argument above, the only terms that are relevant are those where the underlying tree $t$ is expanded. Fix such an expansion, where the vertex $v_{0}$ of $t$ gets expanded into two vertices $v_{1}$ and $v_{2}$, connected by an edge. Call this expanded tree $t^{e}$. Then if $v$ is a vertex of $t$ that is not $v_{0}, \partial \alpha_{v}(t)$ contains one term, $\alpha_{v}\left(t^{e}\right)$, corresponding to the fixed expansion $t^{e}$. On the other hand $\partial \alpha_{v_{0}}(t)$ contains two such terms: $\alpha_{v_{1}}\left(t^{e}\right)$ and $\alpha_{v_{2}}\left(t^{e}\right)$. So for every internal vertex of the expanded tree $t^{e}$, there is exactly one summand where the root attaches to it. Thus $\partial \eta_{\bullet}(t)=\sum \eta_{\bullet}\left(t^{e}\right)$ where the sum is over all expansions of $t$, and so by definition $\partial \eta_{\bullet}(t)=\eta_{\bullet} \partial(t)$. The extra factor of $(-1)^{\operatorname{deg}(t)}$ in the definition of $\eta$ is designed to make the orientations in this equation agree.

\subsection{On the rational homology}

Although not necessary for the main results of this paper, the following proposition confirms that rationally all the homology is concentrated in degree 0 , and gives a crude estimate for the torsion.

Proposition $2.6 \quad(1) \quad H_{k}\left(\mathbb{L}_{\bullet, n} ; \mathbb{Z}\right)$ is $n$ !-torsion, and so $H_{k}\left(\mathbb{L}_{\bullet, n} ; \mathbb{Q}\right)=0$, for all $k \geq 1$.

(2) $\quad H_{k}\left(\mathbb{T}_{\bullet, n+2} ; \mathbb{Z}\right)$ is $(n+2)$ !-torsion, and so $H_{k}\left(\mathbb{T}_{\bullet, n+2} ; \mathbb{Q}\right)=0$, for all $k \geq 1$.

Proof Consider the tree complex $\mathbb{L}_{\bullet, n}^{\dagger}$ defined analogously to $\mathbb{L}_{\bullet, n}$ except that the leaves are always labeled by $1, \ldots, n$ without repeats. This actually corresponds to an augmented cochain complex for a simplicial complex $K_{n}$ defined in the following way. Every tree, except the unique one with only one internal vertex, corresponds to a simplex, given by putting nonnegative lengths summing to 1 on all of its internal edges. (When an edge has length 0 , it contracts to a point.) So we have the isomorphism $\widetilde{H}^{i}\left(K_{n}\right) \cong$ $H_{n-i-3}\left(\mathbb{L}_{\bullet, n}^{\dagger}\right)$, where the index shift comes from the fact that the dimension $i$ of a simplex is one less than the number of internal edges of the corresponding tree, and 
the reader may verify that this corresponds to homological degree $n-i-3$. This simplicial complex $K_{n}$, also known as the Whitehouse complex, is well-known to be homotopy equivalent to a wedge of $(n-1)$ ! spheres of dimension $n-3$ (which is the top dimension). See Readdy [31] for an elementary proof based on discrete Morse theory. In particular, this implies that $H_{k}\left(\mathbb{L}_{\bullet, n}^{\dagger}\right)=0$ for all $k \geq 1$. Let $V=\mathbb{Z}^{m}$ be the abelian group spanned by the label set. The symmetric group $\mathcal{S}_{n}$ acts on $\mathbb{L}_{\bullet, n}^{\dagger}$ by permuting the labels, and we have an isomorphism with the space of coinvariants

$$
\mathbb{L}_{\bullet, n}=\left[V^{\otimes n} \otimes \mathbb{L}_{\bullet, n}^{\dagger}\right]_{\mathcal{S}_{n}},
$$

where $\mathcal{S}_{n}$ acts simultaneously on $\mathbb{L}_{\bullet, n}^{\dagger}$ and $V^{\otimes n}$. Now, if $i>0, H_{i}\left(V^{\otimes n} \otimes \mathbb{L}_{\bullet, n}^{\dagger}\right) \cong$ $V^{\otimes n} \otimes H_{i}\left(\mathbb{L}_{\bullet, n}^{\dagger}\right)=0$. The proof is finished by noting that if a finite group $G$ acts on a chain complex $C_{\bullet}$, where $H_{i}\left(C_{\bullet}\right)=0$, then $H_{i}\left(\left[C_{\bullet}\right]_{G}\right)$ is $|G|$-torsion. To see this note that we have a sequence

$$
\left[C_{\bullet}\right]_{G} \rightarrow C_{\bullet} \rightarrow\left[C_{\bullet}\right]_{G},
$$

where the first map is the map $\sigma \mapsto \sum_{g \in G} g \cdot \sigma$, and the second map is the natural quotient. Their composition is $|G| \cdot \mathrm{Id}$. Applying the functor $H_{i}(\cdot)$, we have $|G| \cdot \operatorname{Id}$ factoring through 0 , implying that $H_{i}\left(\left[C_{\bullet}\right]_{G}\right)$ is $|G|$-torsion.

The proof for $\mathbb{T}_{\bullet, n+2}$ is similar, but one needs to mod out by the action of $\mathcal{S}_{n+2}$, and thereby include the root, instead.

To see that these complexes have trivial rational homology for $k \geq 1$ one cannot appeal to the universal coefficient theorem, which only holds for free chain complexes, but it is still a straightforward exercise to show that for any chain complex $C_{\bullet}$. we have $H_{*}\left(C_{\bullet} ; \mathbb{Q}\right) \cong H_{*}\left(C_{\bullet}\right) \otimes \mathbb{Q}$.

\section{Discrete Morse theory for chain complexes}

In order to prove Theorem 1.4 and Theorem 1.6, we adopt a convenient general framework for constructing quasi-isomorphisms based on Forman's theory of discrete vector fields on simplicial complexes [9]. In the setting we require, this has been studied by Kozlov [21], who proves that the Morse complex, defined below, yields isomorphic homology, but does not construct a map to the Morse complex. We give an elaboration of his proof which has the added benefit of constructing a map to the Morse complex, but we claim no originality.

We start by considering, like Kozlov, chain groups which are free modules over a commutative ring. We then analyze a specific nonfree case: when the chain groups are 
direct sums of copies of $\mathbb{Z}$ and $\mathbb{Z}_{2}$. This analysis can be generalized to other nonfree cases, but we limit ourselves to what we need in this paper.

Definition 3.1 (Homological vector field) Fix a commutative ground $\operatorname{ring} R$, with unit, and suppose that $\left(C_{\bullet}, \partial\right)$ is a chain complex where each $C_{k}$ is a free $R$-module, with a fixed basis $\left\{\mathbf{u}_{\mathbf{k}}^{\mathbf{i}}\right\}$.

(1) A vector is a pair of basis elements $\left(\mathbf{u}_{\mathbf{k}-\mathbf{1}}^{\mathbf{i}}, \mathbf{u}_{\mathbf{k}}\right)$ in degrees $k-1$ and $k$ respectively, such that $\partial\left(\mathbf{u}_{\mathbf{k}}\right)=r_{i} \mathbf{u}_{\mathbf{k}-\mathbf{1}}^{\mathbf{i}}+\sum_{j \neq i} c_{j} \mathbf{u}_{\mathbf{k}-\mathbf{1}}^{\mathbf{j}}$, where $r_{i} \in R$ is invertible, and the coefficients $c_{j} \in R$ are arbitrary.

(2) A (homological) vector field, $\Delta$, is a collection of vectors (a, b) such that every basis element appears in at most one vector of $\Delta$.

(3) A basis element is said to be critical if does not appear in any vector of the vector field $\Delta$. The set of all critical basis elements for $\Delta$ will be denoted $\mathfrak{X}^{\Delta}$.

(4) Given a vector field, $\Delta$, a gradient path is a sequence of basis elements

$$
\mathbf{a}_{1}, \mathbf{b}_{1}, \mathbf{a}_{2}, \mathbf{b}_{2}, \ldots, \mathbf{a}_{\mathbf{m}}
$$

where each $\left(\mathbf{a}_{\mathbf{i}}, \mathbf{b}_{\mathbf{i}}\right) \in \Delta$, and $\mathbf{a}_{\mathbf{i}}$ has nonzero coefficient in $\partial \mathbf{b}_{\mathbf{i}-\mathbf{1}}$ and $\mathbf{a}_{\mathbf{i}} \neq \mathbf{a}_{\mathbf{i}-\mathbf{1}}$. It is often useful to visualize gradient paths using a "zigzag" diagram like the one below.

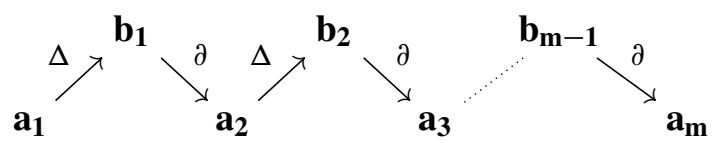

The set of all gradient paths from $\mathbf{a}$ to $\mathbf{a}^{\prime}$ (that is with $\mathbf{a}_{1}=\mathbf{a}$ and $\mathbf{a}_{\mathbf{m}}=\mathbf{a}^{\prime}$ ) is denoted $\Gamma\left(\mathbf{a}, \mathbf{a}^{\prime}\right)=\Gamma^{\Delta}\left(\mathbf{a}, \mathbf{a}^{\prime}\right)$.

(5) A d-gradient path is a sequence of basis elements

$$
\mathbf{b}_{\mathbf{0}}, \mathbf{a}_{1}, \mathbf{b}_{1}, \ldots, \mathbf{a}_{\mathbf{m}}
$$

where $\mathbf{a}_{\mathbf{1}}, \mathbf{b}_{\mathbf{1}}, \cdots, \mathbf{a}_{\mathbf{m}}$ is a gradient path and $\mathbf{a}_{\mathbf{1}}$ has nonzero coefficient in $\partial \mathbf{b}_{\mathbf{0}}$. The appropriate zigzag here is

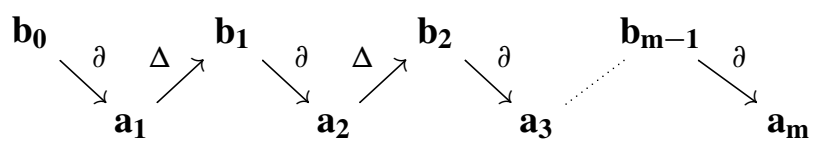

The set of all $\partial$-gradient paths from $\mathbf{b}$ to $\mathbf{a}$ will be denoted $\Gamma_{\partial}(\mathbf{b}, \mathbf{a})=\Gamma_{\partial}^{\Delta}(\mathbf{b}, \mathbf{a})$.

(6) A vector field is said to be a gradient vector field if there are no closed gradient paths. 
Given a vector field, one can construct a degree 1 homomorphism of the same name $\Delta: C_{\bullet} \rightarrow C_{\bullet+1}$ as follows. If $\mathbf{a}$ is a basis element appearing in a vector $(\mathbf{a}, \mathbf{b})$, define $\Delta(\mathbf{a})=\mathbf{b}$, and define $\Delta$ to be zero on all other basis elements.

There are functionals on gradient paths and $\partial$-gradient paths

$$
w: \Gamma\left(\mathbf{a}, \mathbf{a}^{\prime}\right) \rightarrow R \text { and } w: \Gamma_{\partial}(\mathbf{b}, \mathbf{a}) \rightarrow R
$$

called the weight. The weight of a gradient path $\gamma=\left(\mathbf{a}_{1}, \mathbf{b}_{\mathbf{1}}, \mathbf{a}_{\mathbf{2}}, \mathbf{b}_{\mathbf{2}}, \ldots, \mathbf{a}_{\mathbf{m}}\right)$ is defined as follows. For each $\mathbf{a}_{\mathbf{i}}$, where $i>1$, suppose that $\partial \mathbf{b}_{\mathbf{i}-1}=r_{i-1} \mathbf{a}_{\mathbf{i}-\mathbf{1}}+c_{i-1} \mathbf{a}_{\mathbf{i}}+\cdots$, for $r_{i-1}, c_{i-1} \in R$, with $r_{i-1}$ invertible. Define the weight to be

$$
w_{\gamma}=(-1)^{m-1} \frac{c_{1} \cdots c_{m-1}}{r_{1} \cdots r_{m-1}} \in R .
$$

The weight of a $\partial$-gradient path $\mu$ is also multiplied by the coefficient $c_{0}$ of $\mathbf{a}_{\mathbf{1}}$ in $\partial \mathbf{b}_{\mathbf{0}}$ :

$$
w_{\mu}=(-1)^{m-1} \frac{c_{0} c_{1} \cdots c_{m-1}}{r_{1} \cdots r_{m-1}} \in R
$$

We now define the Morse complex $C_{\bullet}^{\Delta}$ for a gradient vector field $\Delta$. The chain groups of $C_{\bullet}^{\Delta}$ are the submodules of $C_{\bullet}$ spanned by critical basis elements. The boundary operator $\partial^{\Delta}$ is defined as follows. Suppose $\mathbf{b} \in \mathfrak{X}^{\Delta}$.

$$
\partial^{\Delta}(\mathbf{b})=\sum_{\mathbf{a} \in \mathfrak{X}^{\Delta}} d_{b a} \mathbf{a}
$$

where

$$
d_{b a}=\sum_{\gamma \in \Gamma_{\partial}(\mathbf{b}, \mathbf{a})} w_{\gamma} .
$$

There is a map $\phi=\phi^{\Delta}:\left(C_{\bullet}, \partial\right) \rightarrow\left(C_{\bullet}^{\Delta}, \partial^{\Delta}\right)$ defined as follows. If $\mathbf{a}$ is critical, then $\phi(\mathbf{a})=\mathbf{a}$. Otherwise

$$
\phi(\mathbf{a})=\sum_{\mathbf{a}^{\prime} \in \mathfrak{X}^{\Delta}} c_{a a^{\prime}} \mathbf{a}^{\prime},
$$

where

$$
c_{a a^{\prime}}=\sum_{\gamma \in \Gamma\left(\mathbf{a}, \mathbf{a}^{\prime}\right)} w_{\gamma}
$$

In particular, it follows from these definitions that for any $\mathbf{a}, \phi(\Delta(\mathbf{a}))=0$.

The map $\phi$ is in some sense defined to be the flow along a vector field. Clearly $\partial^{\Delta}=\phi^{\Delta} \partial$.

Theorem 3.2 The Morse complex is a chain complex: $\left(\partial^{\Delta}\right)^{2}=0$, and $\phi^{\Delta}$ is a chain map which induces an isomorphism $H_{*}\left(C_{\bullet}\right) \stackrel{\cong}{\rightrightarrows} H_{*}\left(C_{\bullet}^{\Delta}\right)$. 
Proof We prove all statements simultaneously by induction on the number of vectors in the vector field. The base case of our analysis will be one vector, say $\Delta(\mathbf{a})=\mathbf{b}$, and $\partial \mathbf{b}=r \mathbf{a}+\cdots$. Consider the acyclic subcomplex $A=(0 \rightarrow R(\mathbf{b}) \rightarrow R(\partial \mathbf{b}) \rightarrow 0)$. We get a quasi-isomorphism $C_{\bullet} \rightarrow C_{\bullet} / A$. Now as a free $R$-module, we have an isomorphism $C_{\bullet} / A \cong C_{\bullet}^{\Delta}$ under the map defined on generators $[\mathbf{b}] \mapsto 0,[\mathbf{a}] \mapsto \mathbf{a}-\frac{1}{r} \partial \mathbf{b}$, and $[\mathbf{c}] \mapsto \mathbf{c}$ otherwise. The gradient paths in $C_{\bullet}$ are all of the form $\mathbf{a}, \mathbf{b}, \mathbf{c}$ where $\mathbf{c}$ is a nonzero term in $\partial \mathbf{b}$ not equal to $\mathbf{a}$. The weight of this path is the negative of the coefficient of $\mathbf{c}$ in $\partial \mathbf{b}$, divided by $r$. Hence $\phi(\mathbf{a})=-\frac{1}{r} \partial \mathbf{b}+\mathbf{a}$. On the other hand $\phi(\mathbf{b})=0$ and $\phi(\mathbf{c})=\mathbf{c}$ for critical generators. So the isomorphism is given by $\phi$ as claimed.

The boundary $\partial$ on $C_{\bullet} / A$ induces the boundary operator $\phi \partial \phi^{-1}$ on $C_{\bullet}^{\Delta}$, and we must now determine its form. Consider a critical generator $\mathbf{c}$. We have that $\phi^{-1}(\mathbf{c})=[\mathbf{c}]$. So $\phi \partial \phi^{-1}(\mathbf{c})=\phi \partial \mathbf{c}=\partial^{\Delta} \mathbf{c}$ as desired.

Now suppose the theorem is true for gradient vector fields with $k$ vectors, and assume we now have one with $k+1$ vectors. Choose a vector, $\mathbf{b}=\Delta \mathbf{a}$.

Let $\Delta_{k}$ be the vector field consisting of the $k$ vectors aside from $(\mathbf{a}, \mathbf{b})$. Inductively we have a quasi-isomorphism

$$
\phi^{k}:=\phi^{\Delta_{k}}:\left(C_{\bullet}, \partial\right) \rightarrow\left(C_{\bullet}^{\Delta_{k}}, \partial^{\Delta_{k}}\right) .
$$

Now, we claim the pair $(\mathbf{a}, \mathbf{b})$ still represents a vector in this Morse complex. We need only verify that $\partial^{\Delta_{k}} \mathbf{b}=r \mathbf{a}+\cdots$. This follows from the nonexistence of closed gradient paths in the original vector field, since a $\partial$-gradient path from $\mathbf{b}$ to $\mathbf{a}$ aside from the path $(\mathbf{b}, \mathbf{a})$ would combine with the vector $(\mathbf{a}, \mathbf{b})$ to form a closed gradient path. Let $\Delta_{1}$ be the vector field with the single vector $(\mathbf{a}, \mathbf{b})$ on the Morse complex $C_{\bullet}^{\Delta_{k}}$. Then $\left(C_{\bullet}^{\Delta_{k}}\right)^{\Delta_{1}}=C_{\bullet}^{\Delta}$. So we also have a quasi-isomorphism

$$
\phi^{1}:=\phi^{\Delta_{1}}:\left(C_{\bullet}^{\Delta_{k}}, \partial^{\Delta_{k}}\right) \rightarrow\left(C_{\bullet}^{\Delta}, \partial^{\Delta_{1}}\right),
$$

where we emphasize that $\partial^{\Delta_{1}}$ is defined to be the weighted sum of $\partial$-gradient paths alternating between $\partial^{\Delta_{k}}$ and $\Delta_{1}$. We now need to check that $\phi^{\Delta}=\phi^{1} \phi^{k}$, and that $\partial^{\Delta_{1}}=\partial^{\Delta}$. In the following calculations let $\mathfrak{X}^{i}=\mathfrak{X}^{\Delta_{i}}$, and let $\mathfrak{X}=\mathfrak{X}^{\Delta}$.

$$
\begin{aligned}
\phi^{1} \phi^{k}(\mathbf{u}) & =\phi^{1} \sum_{\mathbf{v} \in \mathfrak{X}^{k}} c_{u v}^{k} \mathbf{v} \\
& =\sum_{\mathbf{v} \in \mathfrak{X}^{k}} \sum_{\mathbf{w} \in \mathfrak{X}} c_{u v}^{k} c_{v w}^{1} \mathbf{w},
\end{aligned}
$$

where $c_{u v}^{i}$ measures gradient paths with respect to $\Delta_{i}$. So we need to check that

$$
\sum_{\mathbf{v} \in \mathfrak{X}^{k}} c_{u v}^{k} c_{v w}^{1}=c_{u w} \quad \text { for all } \mathbf{w} \in \mathfrak{X}
$$


Note that $\mathfrak{X}=\mathfrak{X}^{k} \backslash\{\mathbf{a}, \mathbf{b}\}$, so we can write

$$
\begin{aligned}
\sum_{\mathbf{v} \in \mathfrak{X}^{k}} c_{u v}^{k} c_{v w}^{1} & =\left(\sum_{\mathbf{v} \in \mathfrak{X}} c_{u v}^{k} c_{v w}^{1}\right)+c_{u b}^{k} c_{b w}^{1}+c_{u a}^{k} c_{a w}^{1} \\
& =c_{u w}^{k}+c_{u a}^{k} c_{a w}^{1} .
\end{aligned}
$$

The first term simplifies as indicated, because when $\mathbf{v}, \mathbf{w} \in \mathfrak{X}, c_{v w}^{1}$ is only nonzero if $\mathbf{v}=\mathbf{w}$. The term $c_{b w}^{1}$ is zero because $\mathbf{b}$, being $\Delta_{1}(\mathbf{a})$, does not begin any gradient paths. The term $c_{u w}^{k}$ measures gradient paths from $\mathbf{u}$ to $\mathbf{w}$ which do not involve the vector $(\mathbf{a}, \mathbf{b}) . c_{u a}^{k}$ measures all gradient paths that end in $\mathbf{a}$, and $c_{a w}^{1}$ represents all gradient paths (alternating between $\Delta_{1}$ and $\partial^{\Delta_{k}}$ ) from a to $\mathbf{w}$. There is only one vector in $\Delta_{1}$, and $\partial^{\Delta_{k}}$ measures alternating paths between $\partial$ and $\Delta_{k}$, so $c_{a w}^{1}$ represents all gradient paths with respect to $\Delta$ that start with the basis element a and end at $\mathbf{w}$. So the product $c_{u a}^{k} c_{a w}^{1}$ measures all gradient paths that pass through the basis element $\mathbf{a}$. Thus the sum measures all gradient paths from $\mathbf{u}$ to $\mathbf{w}: c_{u w}^{k}+c_{u a}^{k} c_{a w}^{1}=c_{u w}$.

The equations $\partial^{\Delta_{k}}=\phi^{k} \partial$ and $\partial^{\Delta_{1}}=\phi^{1} \partial^{\Delta_{k}}$ imply that $\partial^{\Delta_{1}}=\phi^{1} \phi^{k} \partial=\phi \partial=\partial^{\Delta}$.

\subsection{Nonfree chain complexes}

We will need to adapt the above construction to chain complexes which are not free. Indeed our tree complexes all have 2-torsion, so we adapt the notion of a gradient vector field to the case where the chain groups consist of both $\mathbb{Z}$ - and $\mathbb{Z}_{2}$-summands. We replace the notion of "basis of a free $R$-module" with the notion of "minimal generating set," where some generators span copies of $\mathbb{Z}$ and some span copies of $\mathbb{Z}_{2}$. We define a vector field as above except that a vector $(\mathbf{a}, \mathbf{b})$ cannot mix a $\mathbb{Z}_{2}$-generator and a $\mathbb{Z}$-generator. Then $C_{\bullet}^{\Delta}$ is defined to be the subgroup of the chain group $C_{\bullet}$ spanned by critical generators. (In particular, $\mathbb{Z}_{2}$-generators remain 2 -torsion in the Morse complex.) Gradient paths are defined as above, and come in two types. A gradient path ending in $\mathbf{a}$ is called a $\mathbb{Z}_{2}$-path if $\mathbf{a}$ is a $\mathbb{Z}_{2}$-generator and is called a $\mathbb{Z}$-path if $\mathbf{a}$ is a $\mathbb{Z}$-generator. Notice that if a gradient path involves a $\mathbb{Z}_{2}$-generator at some stage, then every subsequent generator in the path will be a $\mathbb{Z}_{2}$-generator. In particular, a $\mathbb{Z}$-path will only consist of $\mathbb{Z}$-generators. The weight of a gradient path is defined as follows. It is defined by the same formula as in the free case for $\mathbb{Z}$-paths:

$$
w_{\gamma}=(-1)^{m-1} \frac{c_{1} \cdots c_{m-1}}{r_{1} \cdots r_{m-1}} \in \mathbb{Z} .
$$

For $\mathbb{Z}_{2}$-paths, $w_{\gamma}=c_{1} \cdots c_{m-1} \in \mathbb{Z}_{2}$, where some of these coefficients may be in $\mathbb{Z}$, but are interpreted mod 2. The $r_{i}$ 's are omitted as they are all \pm 1 , so are irrelevant mod 2. The weights of $\partial$-gradient paths are defined similarly. 
The flow $\phi^{\Delta}$ is defined as a sum of weights of gradient paths, as in the free case. With these definitions, the proof of Theorem 3.2 then goes through with little modification in this particular nonfree setting:

Theorem 3.3 Let $C$ • be a chain complex where each $C_{k}$ is a finitely generated abelian group whose only torsion is 2-torsion, and suppose that $\Delta$ is a gradient vector field in the above sense on $C_{\bullet}$. Then the Morse complex is a chain complex: $\left(\partial^{\Delta}\right)^{2}=0$, and $\phi^{\Delta}$ is a chain map which induces an isomorphism $H_{*}\left(C_{\bullet}\right) \stackrel{\cong}{\rightrightarrows} H_{*}\left(C_{\bullet}^{\Delta}\right)$.

Sketch of proof Given a vector $(\mathbf{a}, \mathbf{b})$ proceed as in Theorem 3.2 by modding out by an acyclic complex $0 \rightarrow\langle\mathbf{b}\rangle \rightarrow\langle\partial \mathbf{b}\rangle \rightarrow 0$, where the notation $\langle x\rangle$ indicates the subgroup generated by $x$. If $\mathbf{a}, \mathbf{b}$ are both $\mathbb{Z}$-generators, then the associated complex is isomorphic to the acyclic complex $0 \rightarrow \mathbb{Z} \rightarrow \mathbb{Z} \rightarrow 0$ because of the presence of the $\mathbb{Z}$-generator $\mathbf{a}$ in $\partial \mathbf{b}$. If they are both $\mathbb{Z}_{2}$-generators, then it is isomorphic to $0 \rightarrow \mathbb{Z}_{2} \rightarrow \mathbb{Z}_{2} \rightarrow 0$. Thus the base case of the proof is established, and the rest of the proof of Theorem 3.2 can be copied verbatim.

\section{Proof of Theorem 1.6}

Recall the statement of Theorem 1.6: $H_{1}\left(\mathbb{L}_{\bullet, n} ; \mathbb{Z}\right)=0$. The proof is done in two stages by Propositions 4.1 and 4.2, whose proofs occupy most of this section.

Let $\mathbb{L}_{\bullet, n}^{(2)} \subset \mathbb{L}_{\bullet, n}$ be the subcomplex spanned by $2 \mathbb{L}_{\bullet, n}$ and degree 2 trees which have two 4-valent vertices which are swapped by some automorphism, as illustrated below, and such that there is no orientation-reversing symmetry.

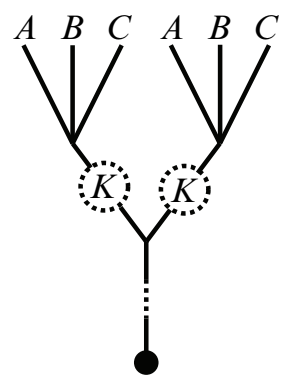

(The obvious symmetry exchanging the two 4-valent vertices is orientation preserving, because the trees being swapped have an even number of edges. See the proof of Proposition 4.1 for more discussion.) This is evidently a subcomplex since the boundary of such a tree is a multiple of 2 . 
Proposition 4.1 For all $n, H_{1}\left(\mathbb{L}_{\bullet, n} ; \mathbb{Z}\left[\frac{1}{2}\right]\right)=0$, and $H_{1}\left(\mathbb{L}_{\bullet, n}^{(2)} ; \mathbb{Z}\right)=0$.

Proposition 4.2 For all $n, H_{1}\left(\mathbb{L}_{\bullet, n} ; \mathbb{Z}_{2}\right)=0$.

Before proving these propositions, we check that they imply Theorem 1.6. We would like to use the universal coefficient theorem to conclude that $H_{1}\left(\mathbb{L}_{\bullet, n} ; \mathbb{Z}\right)=0$, but our chain groups are not free modules, so we pause to state a lemma that holds in this context. For any chain complex $C \bullet$ define

$$
H_{k}^{(2)}\left(C_{\bullet}\right)=\frac{Z_{k} \cap 2 C_{k}}{2 Z_{k}+\left(B_{k} \cap 2 C_{k}\right)},
$$

where $Z_{k}$ and $B_{k}$ are the submodules of cycles and boundaries, respectively.

Lemma 4.3 For all $k$, there is an exact sequence

$$
0 \rightarrow H_{k}^{(2)}\left(C_{\bullet}\right) \rightarrow \mathbb{Z}_{2} \otimes H_{k}\left(C_{\bullet}\right) \rightarrow H_{k}\left(C_{\bullet} ; \mathbb{Z}_{2}\right) .
$$

Proof The right-hand map is defined by $1 \otimes[z] \mapsto[1 \otimes z]$ for cycles $z$. Clearly $[1 \otimes z]$ is still a cycle. This is well-defined because boundaries $1 \otimes \partial w$ map to boundaries $\partial(1 \otimes w)$. We claim that the left-hand map is an injection. Suppose a cycle $2 u$ maps to 0 in $\mathbb{Z}_{2} \otimes H_{k}\left(C_{\bullet}\right)$. So $1 \otimes 2 u=\partial(1 \otimes w)$, implying $1 \otimes 2 u=1 \otimes \partial w$ in $\mathbb{Z}_{2} \otimes C_{\bullet}$. Therefore, $\partial w=2 u+2 z$, where $z$ is a cycle. Therefore $2 u \in 2 Z_{k}+\left(B_{k} \cap 2 C_{k}\right)$, and hence equals 0 in the domain. Finally, to see exactness at the middle, suppose that $1 \otimes[z] \mapsto 0 \in H_{k}\left(C_{\bullet} ; \mathbb{Z}_{2}\right)$. Then $1 \otimes z=\partial(1 \otimes w)$. Therefore $\partial w=z+2 x$. Hence $z-\partial w \in 2 C_{\bullet}$, and the homology class $[z]=[z-\partial w]$ is represented by a cycle in $2 C_{\bullet}$.

Proof of Theorem 1.6 We apply Lemma 4.3, using Propositions 4.1 and 4.2: Notice first that $H_{1}\left(\mathbb{L}_{\bullet, n}^{(2)} ; \mathbb{Z}\right)$ surjects onto $H_{1}^{(2)}\left(\mathbb{L}_{\bullet, n} ; \mathbb{Z}\right)$, because $\mathbb{L}_{1, n}^{(2)}=2 \mathbb{L}_{1, n}$. Thus $\mathbb{Z}_{2} \otimes H_{1}\left(\mathbb{L}_{\bullet, n} ; \mathbb{Z}\right)$ is trapped between two zero groups and is therefore zero. And tensor-

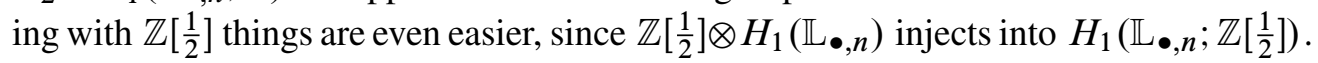

\subsection{Plan of the proofs of Propositions 4.1 and 4.2}

Because the proofs of these two propositions are somewhat technical, we would like to give an overview of the structure of these proofs. Fix $n$, and to simplify notation let $\mathbb{L}_{\bullet}=\mathbb{L}_{\bullet, n}$. The idea will be, with two different sets of coefficients, to construct a vector field $\Delta=\Delta_{0} \cup \Delta_{1}$, where $\Delta_{i}: \mathbb{L}_{i} \rightarrow \mathbb{L}_{i+1}$, which has no critical basis elements in degree 1 and then appeal to Theorem 3.2 to conclude the degree 1 homology vanishes. 
First consider $\mathbb{Z}\left[\frac{1}{2}\right]$ coefficients. Since $H_{0}\left(\mathbb{L} \bullet ; \mathbb{Z}\left[\frac{1}{2}\right]\right) \cong \mathbb{Z}\left[\frac{1}{2}\right] \otimes L_{n}$ has a well-known basis, called the Hall basis, our strategy in the $\mathbb{Z}\left[\frac{1}{2}\right]$ case will be to define $\Delta_{0}(J)$ to be some nontrivial contraction of $J$ for every non-Hall tree $J$. In particular, we will define a "Hall problem" of a tree $J$ to be a place in $J$ where two Hall trees meet at a vertex, but their bracket is not itself Hall. $\Delta_{0}(J)$ then contracts an edge at the base of one of the two Hall trees. The resulting vector field has no closed gradient paths, in some sense because the Hall basis algorithm "works." The way we rigorously prove it is to show that a natural "Hall order" defined on trees always increases as one moves along a gradient path. This is the most one can hope to do for $\Delta_{0}$, because Hall trees need to survive as a basis for $H_{0}\left(\mathbb{L} \bullet ; \mathbb{Z}\left[\frac{1}{2}\right]\right)$.

All the trees in the image of $\Delta_{0}$ are not critical, so it suffices now to define $\Delta_{1}$ to be nonzero on all the other degree 1 trees. In analogy with degree 0 , we say a tree is Hall $1_{1}$ if it is in the image of $\Delta_{0}$, because these are the trees on which $\Delta_{1}$ needs to vanish. We combinatorially characterize what it means to be Hall 1 , and define a "Hall 1 problem" to be one of an exhaustive list of ways that a tree can fail to be Hall 1 . Finally we define $\Delta_{1}$ for each different type of Hall 1 problem as a certain contraction of an edge within the Hall 1 problem. The resulting vector field is again shown to be gradient by arguing that the Hall order increases as one moves along gradient paths. This then proves the $\mathbb{Z}\left[\frac{1}{2}\right]$ case, and in fact proves Proposition 4.1 since the chain complexes $\mathbb{Z}\left[\frac{1}{2}\right] \otimes \mathbb{L} \bullet$ and $\mathbb{Z} \otimes \mathbb{L}_{\bullet}^{(2)}$ are isomorphic.

For $\mathbb{Z}_{2}$ coefficients the argument is similar, except now we use the fact proven by Levine [24], that $H_{0}\left(\mathbb{L}_{\bullet} ; \mathbb{Z}_{2}\right) \cong \mathbb{Z}_{2} \otimes \mathrm{L}_{n}^{\prime}$ has a basis given by Hall trees plus trees $(H, H)$ where $H$ is Hall. We call the trees in this basis Hall' trees, and proceeding as before, we define $\Delta_{0}^{\prime}$ by contracting Hall ${ }^{\prime}$ problems. Defining a Hall ${ }_{1}^{\prime}$ tree to be a tree in the image of $\Delta_{0}^{\prime}$, a Hall ${ }_{1}^{\prime}$ problem is one of an exhaustive list of ways that a tree can fail to be Hall ${ }_{1}^{\prime}$. Then $\Delta_{1}^{\prime}$ is defined for these different Hall ${ }_{1}^{\prime}$ problems by contracting certain edges within the problems. We argue that the Hall order increases along gradient paths, except in one case, and using special arguments to take care of this case, this shows that there are no closed gradient paths.

Remark It may seem as though Theorem 3.3 should be used to construct a single vector field directly on $\mathbb{L}_{\bullet, n}$ instead of constructing two different vector fields with $\mathbb{Z}\left[\frac{1}{2}\right]$ - and $\mathbb{Z}_{2}$-coefficients respectively. This fails because one would need to mod out by acyclic subcomplexes of $\mathbb{L}_{\bullet, n}$ isomorphic to

$$
0 \rightarrow \mathbb{Z} \stackrel{\times 2}{\rightarrow} \mathbb{Z} \rightarrow \mathbb{Z}_{2} \rightarrow 0
$$

which are not part of the vector field formalism. Indeed the degree 2 tree depicted at the beginning of Section 4 sits inside a subcomplex of this form: 


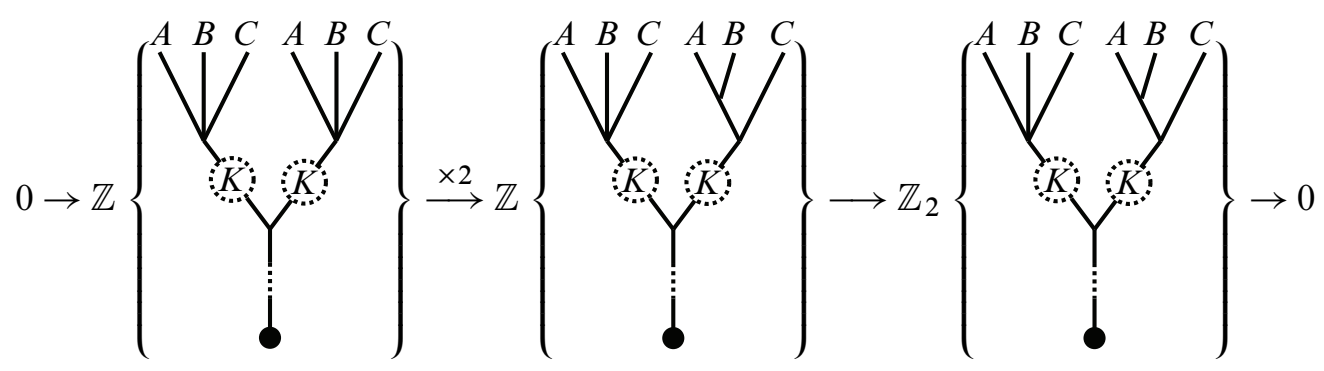

With $\mathbb{Z}\left[\frac{1}{2}\right]$-coefficients, we will pair the two trees on the left as a vector, and with $\mathbb{Z}_{2}$-coefficients the two trees on the right will be paired, allowing us to kill the degree 1 tree in the middle in both cases. With $\mathbb{Z}$-coefficients the degree 1 tree in the middle cannot be part of a vector, so would necessarily survive to the Morse complex.

\subsection{Proof of Proposition 4.1}

We prove both cases simultaneously. Recall that the chain group $\mathbb{L} \bullet$ is defined as the quotient of a free $\mathbb{Z}$-module of oriented trees by relations $(J,-$ or $)=-(J$, or $)$. In particular, a tree $J$ will either generate a $\mathbb{Z}$ - or a $\mathbb{Z}_{2}$-summand depending on whether it has an orientation reversing automorphism, so that $\mathbb{L}_{\bullet}$ is a direct sum of copies of $\mathbb{Z}$ and $\mathbb{Z}_{2}$. We claim that the nonzero trees in $\mathbb{Z}\left[\frac{1}{2}\right] \otimes \mathbb{L} \bullet$ and $\mathbb{L}_{\bullet}^{(2)}$ are the same. Multiplying by 2 and adjoining $1 / 2$ both have the effect of killing the $2-$ torsion, and the additional symmetric trees in $\mathbb{L}_{2}^{(2)}$ are not 2-torsion, so that this is indeed true.

To define a gradient vector field, first we need to specify a basis. Choose orientations for each tree. Define the basis of the free $\mathbb{Z}\left[\frac{1}{2}\right]$-module $\mathbb{Z}\left[\frac{1}{2}\right] \otimes \mathbb{L}$ • to be the trees with specified orientations, except for nonzero symmetric degree 2 trees as specified above and on the right of Figure 5. In that case the basis element is defined to be $1 / 2$ the given oriented tree. For the free $\mathbb{Z}$-module $\mathbb{L}_{\bullet}^{(2)}$, define the basis to be exactly twice the oriented trees just mentioned. In fact $\mathbb{Z}\left[\frac{1}{2}\right] \otimes \mathbb{L} \bullet \cong \mathbb{L}_{\bullet}^{(2)}$ as chain complexes with these specified generators. Thus it makes sense to construct a gradient vector field on both $\mathbb{Z}\left[\frac{1}{2}\right] \otimes \mathbb{L}$ • and $\mathbb{L}_{\bullet}^{(2)}$ simultaneously. We will construct such a vector field with no critical generators in degree 1 . We will work with $\mathbb{Z}\left[\frac{1}{2}\right] \otimes \mathbb{L}$. but since the correspondence of bases respects the boundary operator, this will simultaneously prove the $\mathbb{L}_{\bullet}^{(2)}$ case.

The vector field will consist of pieces $\Delta_{0}: \mathbb{Z}\left[\frac{1}{2}\right] \otimes \mathbb{L}_{0} \rightarrow \mathbb{Z}\left[\frac{1}{2}\right] \otimes \mathbb{L}_{1}$ and $\Delta_{1}: \mathbb{Z}\left[\frac{1}{2}\right] \otimes \mathbb{L}_{1} \rightarrow$ $\mathbb{Z}\left[\frac{1}{2}\right] \otimes \mathbb{L}_{2} . \Delta_{0}$ is constructed via the Hall Basis algorithm for the free Lie algebra. So we need to set up some machinery to explain this. Our presentation follows and expands upon Reutenauer [32].

Recall that for rooted trees $J_{1}$ and $J_{2}$, the rooted tree $\left(J_{1}, J_{2}\right)$ is defined by identifying the roots together to a single vertex, and attaching a new rooted edge to this vertex. 
Extend this notation to $\left(J_{1}, \ldots, J_{k}\right)$, which is defined by identifying the roots of all the trees $J_{i}$ to a single vertex, and attaching a new rooted edge to this vertex. Thus the two trees in Figure 4 are notated $((1,2),(1,2))$ and $((1,2,3),(1,2,3))$, respectively.

If $J_{1}, \ldots, J_{k}$ are oriented, the tree $\left(J_{1}, \ldots, J_{k}\right)$ will be oriented by numbering the root edge first, then the edges of $J_{1}$, followed by the edges of $J_{2}$, etc.

Given a rooted tree, suppose one deletes an internal vertex. Then there are multiple connected components, including the one containing the root. Any of the connected components that does not contain the root can itself be regarded as a rooted tree, by filling in the deleted vertex as a root. Such a tree will be called a full subtree.

With this notation and terminology in hand, we can characterize those rooted trees which have orientation-reversing automorphisms, and are therefore zero when tensoring with $\mathbb{Z}\left[\frac{1}{2}\right]$. Indeed, if a tree contains a full subtree of the form $\left(J_{1}, \ldots, J_{m}\right)$ where $J_{i}=J_{k}$ for some $i \neq k$ with $J_{i}=J_{k}$ having an odd number of edges, then the tree is zero. For example, this will be true when $J_{i}$ is unitrivalent. Hence, over $\mathbb{Z}\left[\frac{1}{2}\right]$ we may assume that for trees in $\mathbb{L}_{0}$ and in $\mathbb{L}_{1}$ the emanating subtrees at every vertex are distinct. Exemplar trees with orientation preserving and reversing automorphisms are pictured in Figure 4.
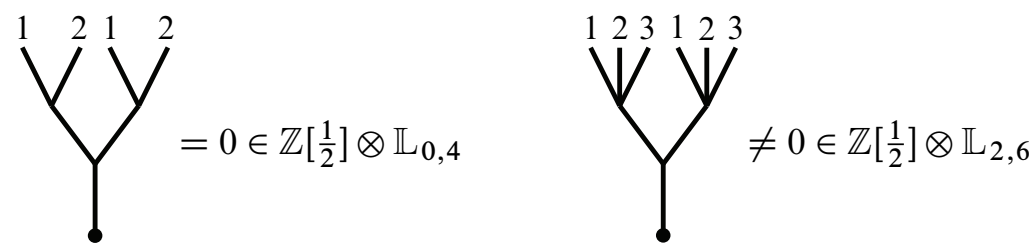

Figure 4 . The tree $((1,2),(1,2))$ on the left has an orientation reversing symmetry, whereas the tree $((1,2,3),(1,2,3))$ on the right has an orientation preserving symmetry.

Definition 4.4 Define the weight of a tree, denoted by $|J|$, to be the number of leaves (the number of univalent vertices not counting the root). We recursively define an order relation, called the Hall order, on (unoriented) labeled trees in the following way.

(1) Trees of weight 1 are ordered by an ordering on the index set.

(2) If $|J|<|K|$, then $J \succ K$.

(3) If $|J|=|K|$ and $J=\left(J_{1}, \ldots, J_{\ell}\right), K=\left(K_{1}, \ldots, K_{m}\right)$ with $\ell<m$ then $J \succ K$. 
(4) If $|J|=|K|$ and $J=\left(J_{1}, \ldots, J_{m}\right), K=\left(K_{1}, \ldots, K_{m}\right)$, assume that $J_{1} \preceq J_{2} \preceq$ $\cdots \preceq J_{m}$ and $K_{1} \preceq K_{2} \preceq \cdots \preceq K_{m}$. Then compare the two trees lexicographically: $J \prec K$ if an only if there is some index $\ell, 1 \leq \ell<m$, such that $J_{i}=K_{i}$ for all $i \leq \ell$ and $J_{\ell+1} \prec K_{\ell+1}$.

Lemma 4.5 Suppose one replaces a full subtree of a tree by a tree with increased Hall order. Then the new tree has larger Hall order than the original.

Proof If the new subtree has lower weight, then this is clear. If the new tree has the same weight, then recursively it will suffice to consider the replacement $\left(J_{1}, \ldots, J_{\ell}, \ldots, J_{m}\right) \mapsto\left(J_{1}, \ldots, J_{\ell}^{\prime}, \ldots, J_{m}\right)$ where the full subtree $J_{\ell}$ has been replaced by $J_{\ell}^{\prime}$, with $J_{\ell} \prec J_{\ell}^{\prime}$. By the lexicographic definition (case (4) of Definition 4.4), the whole tree is also larger with respect to $\prec$.

Definition 4.6 (1) The set of Hall trees is the subset of (unoriented) labeled rooted unitrivalent trees, defined recursively as follows. All weight 1 trees are Hall. The bracket of two Hall trees $\left(H_{1}, H_{2}\right)$ with $H_{1} \prec H_{2}$ is Hall if and only if either $H_{1}$ is of weight 1 , or $H_{1}=\left(H^{\prime}, H^{\prime \prime}\right)$ with $H^{\prime} \prec H^{\prime \prime}$ and $H^{\prime \prime} \succeq H_{2}$.

(2) Given a tree $J$, a Hall problem is a full subtree of $J$ of the form $\left(H_{1}, H_{2}\right)$ where $H_{1} \prec H_{2}$ are both Hall, but $H_{1}=\left(H^{\prime}, H^{\prime \prime}\right)$ with $H^{\prime} \prec H^{\prime \prime} \prec H_{2}$.

(3) The contraction of a Hall problem is the tree obtained by replacing the full subtree $\left(H_{1}, H_{2}\right)$ by the full subtree $\left(H^{\prime}, H^{\prime \prime}, H_{2}\right)$.

The above definitions are made for unoriented trees, but we will often not distinguish between oriented and unoriented trees. For example, when we say that an oriented tree is Hall, we mean that the underlying unoriented tree is Hall.

We now define a vector field $\Delta_{0}: \mathbb{Z}\left[\frac{1}{2}\right] \otimes \mathbb{L}_{0} \rightarrow \mathbb{Z}\left[\frac{1}{2}\right] \otimes \mathbb{L}_{1}$ as follows. If $H$ is a Hall tree, then $\Delta_{0}(H)=0$. Otherwise, suppose $J$ is a tree where there is at least one Hall problem. Define

$\Delta_{0}(J)=\max \left\{J^{c} \mid J^{c}\right.$ is the contraction of a Hall problem in $\left.J\right\}$.

Lemma 4.7 $\Delta_{0}$ is a gradient vector field which is nonzero on any non-Hall tree.

Proof We need to check the following three conditions. Note that condition (2) implies that $\Delta_{0}(J) \neq 0$ if $J \neq 0$, and in particular rules out the possibility that $\Delta_{0}(J)$ has an orientation-reversing automorphism.

(1) $\Delta_{0}\left(J_{1}\right) \neq \Delta_{0}\left(J_{2}\right)$ for distinct $J_{1}, J_{2}$ which are non-Hall.

(2) $\partial \Delta_{0}(J)= \pm J+$ other trees, for $J$ non-Hall.

(3) All gradient paths terminate. 
All three of these will follow from the fact that the two expansions of $\Delta_{0}(J)$, other than $J$ itself, have larger Hall order, which we now check. Indeed, by Lemma 4.5, we just need to show

$$
\left(\left(H^{\prime}, H^{\prime \prime}\right), H_{2}\right) \prec\left(\left(H^{\prime}, H_{2}\right), H^{\prime \prime}\right) \quad \text { and } \quad\left(\left(H^{\prime}, H^{\prime \prime}\right), H_{2}\right) \prec\left(\left(H^{\prime \prime}, H_{2}\right), H^{\prime}\right) .
$$

Note we are assuming that $H^{\prime} \prec H^{\prime \prime} \prec H_{2}$, corresponding to the Hall problem $\left(\left(H^{\prime}, H^{\prime \prime}\right), H_{2}\right)$ in $J$. Then $\left(H^{\prime}, H_{2}\right) \prec H^{\prime} \prec H^{\prime \prime}$, so the term $\left(\left(H^{\prime}, H_{2}\right), H^{\prime \prime}\right)$ is in nondecreasing order. So applying the lexicographic definition, it suffices to show that $\left(H^{\prime}, H^{\prime \prime}\right) \prec\left(H^{\prime}, H_{2}\right)$ which follows from Lemma 4.5. Now for the second inequality above, the two component trees of $\left(\left(H^{\prime \prime}, H_{2}\right), H^{\prime}\right)$ may occur in either order, or even be equal. If they are equal, the tree is zero, and we don't need to consider it, but because we want to reuse this analysis in the case of $\mathbb{Z}_{2}$-coefficients (specifically in the proof of Lemma 4.19), we prefer not to use this fact. In any event, no matter what the order, it suffices to observe that $\left(H^{\prime}, H^{\prime \prime}\right) \prec\left(H^{\prime \prime}, H_{2}\right)$ and $\left(H^{\prime}, H^{\prime \prime}\right) \prec H^{\prime}$. Thus the two expansions of $\Delta_{0}(J)$, other than $J$ itself, have larger Hall order, as claimed.

Condition (1) follows because $\Delta_{0}\left(J_{1}\right)=\Delta_{0}\left(J_{2}\right)$ implies that $J_{2}$ is another expansion of $\Delta_{0}\left(J_{1}\right)$ so that $J_{1} \prec J_{2}$. Symmetrically $J_{2} \prec J_{1}$, which is a contradiction. Condition (2) follows since $\partial \Delta_{0}(J)=J+J^{\prime}+J^{\prime \prime}$ where $J^{\prime}$ and $J^{\prime \prime}$ have increased Hall order. Condition (3) now follows since the Hall order increases as one flows along a gradient path, implying that all gradient paths must terminate.

So far we have adapted the well-known Hall algorithm to the context of homological vector fields. Since the critical generators in degree zero are Hall trees, we have reproduced the standard fact that the free Lie algebra $\mathbb{Z}\left[\frac{1}{2}\right] \otimes \mathrm{L}_{n} \cong H_{0}\left(\mathbb{L} \bullet ; \mathbb{Z}\left[\frac{1}{2}\right]\right)$ is generated by Hall trees. Since these trees actually form a basis, to make further progress in killing degree 1 generators we will need to construct a vector field $\Delta_{1}: \mathbb{Z}\left[\frac{1}{2}\right] \otimes \mathbb{L}_{1} \rightarrow$ $\mathbb{Z}\left[\frac{1}{2}\right] \otimes \mathbb{L}_{2}$, extending $\Delta_{0}$.

Definition 4.8 A nonzero tree, $H \in \mathbb{Z}\left[\frac{1}{2}\right] \otimes \mathbb{L}_{1}$, is said to be $\mathrm{Hall}_{1}$ if $H=\Delta_{0}(J)$ for some tree $J \in \mathbb{Z}\left[\frac{1}{2}\right] \otimes \mathbb{L}_{0}$.

We now characterize $\mathrm{Hall}_{1}$ trees.

Lemma 4.9 A tree $H \in \mathbb{Z}\left[\frac{1}{2}\right] \otimes \mathbb{L}_{1}$ is Hall if $_{1}$ and only if both of the following two conditions hold.

(1) It contains a full subtree $(A, B, C)$, where $A \prec B \prec C$ are all Hall, such that either $|A|=1$, or $A=\left(A^{\prime}, A^{\prime \prime}\right)$ with $A^{\prime} \prec A^{\prime \prime}$ and $A^{\prime \prime} \succeq B$.

(2) The tree obtained by replacing $(A, B, C)$ with $((A, B), C)$ is nonzero, and $H$ is the largest among all contractions of Hall problems of this expanded tree. 
Proof The first condition says exactly that $(A, B, C)$ is the contraction of a Hall problem $((A, B), C)$, using the fact that $(A, B)$ is Hall. So to check whether $H$ is in the image of $\Delta_{0}$ we need only check that it is the maximal contraction of this expanded tree, as per the second statement.

A Hall 1 problem of a nonzero tree $J$, will be defined by negating the characterization of the previous lemma. Cases (1) and (2) of the following Definition 4.10 are the two ways that condition (1) of the previous Lemma can fail, and cases (3) and (4) are the two ways that condition (2) of the previous Lemma can fail.

Definition 4.10 A Hall $_{1}$ problem in a nonzero tree $J \in \mathbb{Z}\left[\frac{1}{2}\right] \otimes \mathbb{L}_{1}$ is defined to be any one of the following situations:

(1) $J$ contains the full subtree $(A, B, C)$ where at least one of $A, B, C$ fails to be Hall. So there is a Hall problem in one of these trees.

(2) $J$ contains the full subtree $(A, B, C)$ where $A \prec B \prec C$ are all Hall, but $A=\left(A^{\prime}, A^{\prime \prime}\right)$ with $A^{\prime} \prec A^{\prime \prime} \prec B$.

(3) $J$ contains a full subtree $(A, B, C)$, where $A \prec B \prec C$ are all Hall, such that either $|A|=1$, or $A=\left(A^{\prime}, A^{\prime \prime}\right)$ with $A^{\prime} \prec A^{\prime \prime}$ and $A^{\prime \prime} \succeq B$; and the expanded tree containing $((A, B), C)$ is nonzero, but $J$ is not the largest of all contractions of the expanded tree.

(4) $J$ contains a full subtree $(A, B, C)$, where $A \prec B \prec C$ are all Hall, such that either $|A|=1$, or $A=\left(A^{\prime}, A^{\prime \prime}\right)$ with $A^{\prime} \prec A^{\prime \prime}$ and $A^{\prime \prime} \succeq B$; but the expanded tree containing $((A, B), C)$ is zero. This means that $((A, B), C)$ occurs in the tree $J$, and there is a symmetry in the expanded tree exchanging this for the $((A, B), C)$ expansion of $(A, B, C)$. Such a $J$ is pictured in the middle of Figure 5, where the expanded tree on the left is 0 .

Now we define the vector field $\Delta_{1}$. This definition is not canonical, as some choices are involved.

Definition 4.11 If a tree is Hall 1 , we define $\Delta_{1}$ to be zero. (As we need to, in order to make $\Delta_{1}$ disjoint from $\Delta_{0}$.) Otherwise, referring to Definition 4.10, we consider the four types of Hall 1 problems:

(1) If $J$ has a type (1) Hall 1 problem, then define $\Delta_{1}(J)$ to contract some Hall problem in one of the trees $\{A, B, C\}$.

(2) If $J$ has a type (2) Hall 1 problem, then define $\Delta_{1}(J)$ to contract the base edge of $A$. 


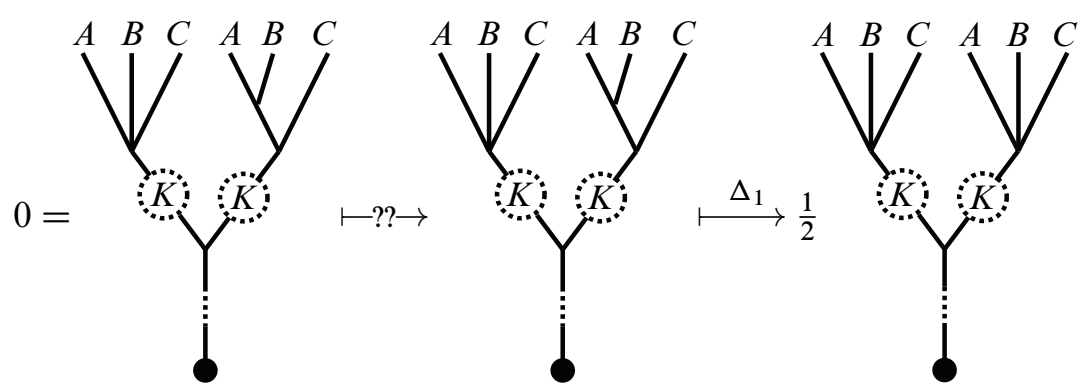

Figure 5. The tree, $J$, in the middle has a type (4) Hall 1 problem, since the expanded tree on the left is equal to zero in $\mathbb{Z}\left[\frac{1}{2}\right] \otimes \mathbb{L}_{0}$. The image of $J$ under $\Delta_{1}$ is pictured on the right. (The sub-trees indicated by the dotted arcs are all trivalent.)

(3) If $J$ has a type (3) Hall 1 problem, to define $\Delta_{1}(J)$, consider the expanded tree $J^{e}$ containing $((A, B), C)$. Then $\Delta_{0}\left(J^{e}\right)$ contracts a different Hall problem in $J^{e}$ (which is maximal among all contractions) than the one that contracts to $J$. Define $\Delta_{1}(J)$ by contracting the image in $J$ of this other Hall problem in $J^{e}$.

(4) If $J$ has a type (4) Hall 1 problem, define $\Delta_{1}(J)$ to be the contraction of the other copy of $((A, B), C)$ with a coefficient of $1 / 2$ (Figure 5 ). This contracted tree has a symmetry, but it is orientation-preserving because it exchanges two trees which have a single $4-$ valent vertex and are otherwise unitrivalent. As mentioned in the introductory remarks to the proof, $1 / 2$ the contracted tree is indeed a basis element.

Lemma 4.12 $\Delta_{1}$ is a gradient vector field which is nonzero on any non-Hall ${ }_{1}$ tree.

Proof As we did for $\Delta_{0}$, we will show that if $J$ is not Hall $1_{1}$, the expansions of $\Delta_{1}(J)$ distinct from $J$ have increased Hall order. So assume $J$ is not Hall 1 . That means there is a Hall 1 problem in $J$.

We analyze the four cases from Definition 4.11 separately:

Case (1) We have a tree $J$ with a full subtree $(A, B, C)$ which has a Hall problem in one of the $A, B, C$ trees. Contracting this Hall problem and then applying $\partial$ without backtracking will either expand the Hall problem into one of the two other trees besides $J$, which we already showed increases the Hall order, or will expand the $(A, B, C)$ vertex, which increases the Hall order because $(A, B, C) \prec(U, V)$ if $|(A, B, C)|=|(U, V)|$. 
Case (2) This case has the most numerous collection of terms. The full subtree $\left(\left(A^{\prime}, A^{\prime \prime}\right), B, C\right)$ with $A^{\prime} \prec A^{\prime \prime} \prec B \prec C$ gets contracted to $\left(A^{\prime}, A^{\prime \prime}, B, C\right)$ and we must show that all terms of $\partial\left(A^{\prime}, A^{\prime \prime}, B, C\right)$ except $\left(\left(A^{\prime}, A^{\prime \prime}\right), B, C\right)$ increase in Hall order. If the 4-valent vertex gets pushed up the tree, like in $\left(\left(A^{\prime}, A^{\prime \prime}, B\right), C\right)$, then the Hall order goes up, so we need only consider the 5 possibilities where this doesn't happen:

$$
\begin{gathered}
\left(\left(A^{\prime}, B\right), A^{\prime \prime}, C\right),\left(\left(A^{\prime}, C\right), A^{\prime \prime}, B\right),\left(\left(A^{\prime \prime}, B\right), A^{\prime}, C\right), \\
\left(\left(A^{\prime \prime}, C\right), A^{\prime}, B\right),\left((B, C), A^{\prime}, A^{\prime \prime}\right)
\end{gathered}
$$

The components of these trees are not necessarily in order, although they definitely are in the first two trees, and in fact the smallest tree in each of the first two triples has larger Hall order than $\left(A^{\prime}, A^{\prime \prime}\right)$. For the third tree, it could be that either of $\left(A^{\prime \prime}, B\right)$ or $A^{\prime}$ is larger, and they could even be equal, however it suffices to observe that both are greater than $\left(A^{\prime}, A^{\prime \prime}\right)$. Similar remarks hold for the fourth tree. For the fifth tree, we know $A^{\prime} \prec A^{\prime \prime}$, but we have no information on $(B, C)$. So it will suffice to observe that $\left(A^{\prime}, A^{\prime \prime}\right) \prec(B, C)$ and $\left(A^{\prime}, A^{\prime \prime}\right) \prec A^{\prime}$.

Case (3) Let $K$ be a nonzero unitrivalent tree with at least two Hall problems, and suppose that $K^{1}$ is the largest contraction of a Hall problem and that $K^{2}$ is the contraction of another one, so that $K^{1} \succeq K^{2}$, and by definition $K^{1}=\Delta_{0}(K)$. Case (3) is the case of the tree $J=K^{2}$, assuming it is distinct from $K^{1}$. Now by definition, $\Delta_{1}\left(K^{2}\right)=K^{12}$, the tree with both Hall problems collapsed. Now we wish to analyze the trees appearing in $\partial\left(K^{12}\right)$ distinct from $K^{2}$. Expanding the first vertex yields the tree $K^{2}$ and two other trees with larger Hall order. Expanding the second vertex yields $K^{1}$ and two other trees larger than $K^{1}$. Since $K_{1} \succ K_{2}$, this shows the Hall order has increased for all of these trees.

Case (4) Here when we expand the tree with two copies of $(A, B, C)$, we will get twice the original tree $J$, which is why we needed to divide by 2 . The other trees are all increased with respect to $\prec$.

Thus we have constructed a gradient vector field $\Delta_{0} \cup \Delta_{1}$ on $\mathbb{Z}\left[\frac{1}{2}\right] \otimes \mathbb{L}$. such that every basis element in degree 1 is either in the range of $\Delta_{0}$ or the support of $\Delta_{1}$. So in the Morse complex, there are no nonzero degree 1 chains, implying the first homology is zero. Incidentally, this also reproduces the classical result that the Hall trees (in degree 0 ) are independent and form a basis for $\mathbb{Z}\left[\frac{1}{2}\right] \otimes L_{n} \cong H_{0}\left(\mathbb{L} \bullet ; \mathbb{Z}\left[\frac{1}{2}\right]\right)$.

This concludes the proof of Proposition 4.1. 


\subsection{Proof of Proposition 4.2}

The proof is very similar to the proof of Proposition 4.1, but now trees with orientationreversing automorphisms are no longer equal to zero in $\mathbb{Z}_{2} \otimes \mathbb{L}$. . We therefore adapt the vector field to take account of these, in some sense generalizing Levine's quasi-Hall basis algorithm [24]. Define the specified basis for $\mathbb{Z}_{2} \otimes \mathbb{L} \bullet$ to consist of unoriented trees. This makes sense because tensoring with $\mathbb{Z}_{2}$ erases the orientation data.

Definition 4.13 (1) A tree in $\mathbb{Z}_{2} \otimes \mathbb{L}$. is a Hall' tree if it is either a Hall tree as defined previously in Definition 4.6 for the $\mathbb{Z}\left[\frac{1}{2}\right]$-coefficients case, or a tree of the form $(H, H)$ where $H$ is a Hall tree.

(2) A Hall problem in a tree $J$ in $\mathbb{Z}_{2} \otimes \mathbb{L} \bullet$ is either a Hall problem as defined in Definition 4.6, or it is a full subtree of $J$ of the form $(H, H)$, for a Hall tree $H$. Call this latter type of problem a symmetric Hall problem.

(3) A Hall' problem in a tree $J$ is a Hall problem which is not of the form $(H, H)$ where $J=(H, H)$ and $H$ is Hall.

(4) The contraction of a Hall' problem is defined as in Definition 4.6, with the contraction of a symmetric Hall problem defined by contracting the root edge of the $(H, H)$ subtree.

These definitions were set up so that

$$
\begin{aligned}
& J \text { is not Hall } \Leftrightarrow J \text { has a Hall problem, } \\
& J \text { is not } \mathrm{Hall}^{\prime} \Leftrightarrow J \text { has a Hall' problem. }
\end{aligned}
$$

Now, similarly to the $\mathbb{Z}\left[\frac{1}{2}\right]$-coefficients case, $\Delta_{0}^{\prime}$ is defined to vanish on Hall' ${ }^{\prime}$ trees; and for $J$ not Hall',$\Delta_{0}^{\prime}(J)$ is defined to be the maximal contraction of a Hall' problem in $J$.

Lemma 4.14 $\Delta_{0}^{\prime}: \mathbb{Z}_{2} \otimes \mathbb{L}_{0} \rightarrow \mathbb{Z}_{2} \otimes \mathbb{L}_{1}$ is a gradient vector field.

Proof As in the proof of Proposition 4.1, we argue that terms in $\partial \Delta_{0}^{\prime}(J)$, other than $J$ itself, have increased Hall order. For Hall' problems which are not symmetric Hall problems, we have already argued this in the proof of Lemma 4.7. For a symmetric Hall problem we contract $((H, H), B)$ to $(H, H, B)$. Notice that $\partial(H, H, B)=$ $2((H, B), H)+((H, H), B)=((H, H), B)$. Thus the only nonzero term in $\partial J$, where $J$ is the tree containing $(H, H, B)$, is the original tree. Thus there are no gradient paths involving the vector $((H, H), B) \mapsto(H, H, B)$.

Definition 4.15 A Hall ${ }_{1}^{\prime}$ tree is defined to be a tree in the image of $\Delta_{0}^{\prime}$. 
Lemma 4.16 A tree $H$ is Hall $_{1}^{\prime}$ if and only if both of the following two conditions hold:

(1) $H$ either

(a) contains a full subtree $(A, B, C)$, where $A \prec B \prec C$ are all Hall, such that either $|A|=1$ or $A=\left(A^{\prime}, A^{\prime \prime}\right)$ with $A^{\prime} \prec A^{\prime \prime}$ and $A^{\prime \prime} \succeq B$;

(b) or it contains a full subtree $(A, A, B)$ where $A$ is Hall.

(2) Let $H^{e}$ be the tree where the full subtree $(A, B, C)$ is expanded to $((A, B), C)$ in case (a) above, or where the full subtree $(A, A, B)$ is expanded to $((A, A), B)$ in case (b). Then $H$ is the largest tree among all contractions of Hall' problems in $H^{e}$.

Proof The first condition characterizes being the contraction of a Hall' problem and the second makes sure that it is a maximal contraction. Because all trees are nonzero in the complex $\mathbb{Z}_{2} \otimes \mathbb{L}_{\bullet}$, there is no longer the subtlety that the expanded tree could be zero (as was the case in Lemma 4.9).

Definition 4.17 Negating the characterization from Lemma 4.16, we define a Hall $_{1}^{\prime}$ problem in a tree $J \in \mathbb{Z}_{2} \otimes \mathbb{L}_{1}$ to be any one of the following situations. Assume $J$ contains the full subtree $(A, B, C)$ with $A \preceq B \preceq C$.

(1) One of the trees $A, B, C$ has a Hall problem:

(a) $A=B$ and $A$ is not Hall.

(b) $A \prec B=C$ and $B$ is not Hall.

(c) $A \prec B \prec C$ and one of $A, B, C$ is not Hall.

(2) $A, B, C$ are Hall with $A \prec B \prec C$ but $A=\left(A^{\prime}, A^{\prime \prime}\right)$ with $A^{\prime} \prec A^{\prime \prime} \prec B$.

(3) $A=B$ are Hall or $B=C$ are Hall or $A \prec B \prec C$ with $|A|=1$ or $A^{\prime \prime} \succeq B$, but this is not the maximal contraction of a Hall' problem in the expanded tree.

Definition 4.18 Define $\Delta_{1}^{\prime}: \mathbb{Z}_{2} \otimes \mathbb{L}_{1} \rightarrow \mathbb{Z}_{2} \otimes \mathbb{L}_{2}$ to vanish on Hall' trees. Otherwise, referring to Definition 4.17, $\Delta_{1}^{\prime}$ is defined for each case as follows:

(1) (a) Define $\Delta_{1}^{\prime}$ to contract some Hall problem in $A$.

(b) Define $\Delta_{1}^{\prime}$ to contract some Hall problem in $B$.

(c) Define $\Delta_{1}^{\prime}$ to contract some Hall problem in $A, B$, or $C$.

Each of these three subcases includes the possibility that the contraction of the Hall problem contracts the root edge of $A, B$ or $C$. For example $A$ might be a tree of the form $(H, H)$, and $\Delta_{1}^{\prime}$ is then defined by contracting the root edge of $A$ to give a full subtree of the form $(H, H, B, C)$. 
(2) As in Definition 4.11, define $\Delta_{1}^{\prime}$ to contract the root edge of $A$.

(3) As in Definition 4.11, define $\Delta_{1}^{\prime}$ to contract the other, maximal, Hall' problem.

Note that as in the definition of $\Delta_{1}$, this definition depends on some choices and has not been made canonical.

Lemma 4.19 $\Delta_{1}^{\prime}: \mathbb{Z}_{2} \otimes \mathbb{L}_{1} \rightarrow \mathbb{Z}_{2} \otimes \mathbb{L}_{2}$ is a gradient vector field.

This will complete the proof of Proposition 4.2, as we have constructed a gradient vector field $\Delta_{0}^{\prime} \cup \Delta_{1}^{\prime}$ on $\mathbb{Z}_{2} \otimes \mathbb{L}$. with no critical basis elements in degree 1 .

We remark that this section gives an independent proof of Levine's result that $\mathbb{Z}_{2} \otimes \mathrm{L}_{n}^{\prime} \cong$ $H_{0}\left(\mathbb{L}_{\bullet} ; \mathbb{Z}_{2}\right)$ is freely generated by Hall' trees.

\subsection{Proof of Lemma 4.19}

We need to verify three things as in the proof of Lemma 4.7:

(1) $\Delta_{1}^{\prime}\left(J_{1}\right) \neq \Delta_{1}^{\prime}\left(J_{2}\right)$ for distinct $J_{1}, J_{2}$ that are not Hall ${ }_{1}^{\prime}$.

(2) $\partial \Delta_{1}^{\prime}(J)=J+$ other trees, for $J$ not Hall' ${ }_{1}^{\prime}$.

(3) All gradient paths terminate.

We proceed similarly to that proof by arguing that in all but one case, the trees in the sum $\partial \Delta^{\prime}(J)-J$ all have larger Hall order than $J$. This exceptional case is dealt with by supplemental arguments that verify the three conditions.

For type (1) Hall ${ }_{1}^{\prime}$ problems, we are contracting a Hall' problem "above" the 4-valent vertex. If this problem is a Hall problem, we have already done the required analysis in the $\mathbb{Z}\left[\frac{1}{2}\right]$-coefficients proof to show that the other expansions have increased Hall order. If it is a contraction of an $(H, H)$ the same analysis applies with the exception of the case singled out in item (1) of the definition of $\Delta_{1}^{\prime}$, namely that the full subtree $((H, H), B, C)$ contracts to $(H, H, B, C)$. For type (2) and (3) Hall $_{1}^{\prime}$ problems, the $\mathbb{Z}\left[\frac{1}{2}\right]$-coefficient analysis remains valid, and the other expansions have increased Hall order.

Now that we know that the Hall order increases along gradient flows in all but the case where the full subtree $((H, H), B, C)$ contracts to $(H, H, B, C)$, we proceed to verify conditions (1), (2) and (3) for this exceptional case. First we check that condition (1) holds. By the proof of Lemma 4.7, the only potential difficulty is when at least one of $J_{1}$ and $J_{2}$ is in the exceptional case. Say $J_{1}$ contains the full subtree $((H, H), B, C)$, which contracts to $(H, H, B, C)$. There are four nonzero terms in $\partial \Delta_{1}^{\prime}\left(J_{1}\right)$ : the 
tree $J_{1}$, and three trees containing the full subtrees $(H, H,(B, C)),((H, H, B), C)$ and $((H, H, C), B)$. With the exception of $J_{1}$, none of these trees contains a Hall ${ }_{1}^{\prime}$ problem that contracts to $(H, H, B, C)$. Thus $(H, H, B, C)$ is the image of a unique tree under $\Delta_{1}^{\prime}$.

Condition (2) also needs only to be checked in the exceptional case, as it automatically follows in the other cases from the fact that the other terms of $\partial \Delta_{1}^{\prime}(J)$ have increased Hall order. So we must verify that the nonzero terms of $\partial(H, H, B, C)$ contain only one copy of $((H, H), B, C)$. The only other term that could be equal to $((H, H), B, C)$ is $(H, H,(B, C))$, which would imply that $B=C=H$. In this case $((H, H), H, H)$ is the contraction of a Hall ${ }^{\prime}$ problem in $((H, H),(H, H))$, and $\Delta_{1}^{\prime}$ is either 0 , if the tree containing $((H, H),(H, H))$ is in the image of $\Delta_{0}^{\prime}$, or $\Delta_{1}^{\prime}$ contracts some other Hall problem elsewhere in the tree. So the hypothesis that $\Delta_{1}^{\prime}$ contracts $((H, H), B, C)$ to $(H, H, B, C)$ is not satisfied.

Finally, we tackle (3). We will show that any gradient path that starts in the exceptional case can never return to its starting point. This implies that a closed gradient path will not contain these exceptional cases, and then the fact that the Hall order increases along gradient paths away from the exceptional cases implies there are no closed paths.

Define a descendant of a tree $T$, to be a tree $S$ such that there is a gradient path starting at $T$ and ending at $S$.

Lemma 4.20 Suppose $\Delta_{1}^{\prime}$ contracts the full subtree $((H, H), B, C)$ to $(H, H, B, C)$. Every descendant of the tree containing $((H, H), B, C)$ is either in the image of $\Delta_{0}^{\prime}$ or contains a full subtree of the form $(H, H, A)$ for some tree $A$.

Corollary 4.21 Any gradient path starting with the tree containing $((H, H), B, C)$ never returns to $((H, H), B, C)$.

Proof of Corollary 4.21 This obviously follows from Lemma 4.20 unless $B=C=H$. However we argued just above in the proof of Lemma 4.19 that this case violates the hypothesis that $\Delta_{1}^{\prime}$ contracts $((H, H), B, C)$ to $(H, H, B, C)$.

Proof of Lemma 4.20 First, consider descendants which are connected by a length 2 gradient path, ie, descendants which are terms of $\partial \Delta^{\prime}-$ id applied to the tree containing $((H, H), B, C)$. There are three such descendants, and they contain trees of the forms

$$
(H, H,(B, C)),((H, H, B), C),((H, H, C), B) .
$$

All three contain a subtree of the appropriate type. So now assume inductively that $J$ is a $k$-th descendant containing a full subtree of the form $(H, H, A)$ and consider what 
trees are connected to $J$ by a length 2 gradient path starting at $J$. Since $(H, H, A)$ is the contraction of a Hall' problem, $\Delta_{1}^{\prime}(J)$ will either be 0 , if it was the maximal contraction, or it will contract a different Hall' problem which gives the maximal contraction, possibly in the tree $A$. So $\Delta_{1}^{\prime}(J)$ contains one of the following subtrees, depending on the nature of the contracted Hall' problem:

(1) $(H, H, A)$ if the Hall' problem giving the maximal contraction is not in $A$.

(2) $\left(H, H, A^{c}\right)$ if the Hall' problem giving the maximal contraction is in $A$ but this is not a contraction of the root. Here $A^{c}$ represents the contraction of this Hall' problem in $A$.

(3) $(H, H, K, K)$ if $A=(K, K)$ and $\Delta_{1}^{\prime}$ contracts the root of $A$.

Applying $\partial$ without backtracking will always yield a subtree $(H, H, B)$ except for the trees containing, respectively, $((H, H), A),\left((H, H), A^{c}\right)$ and $((H, H), K, K)$. However these trees are all in the image of $\Delta_{0}^{\prime}$ as they are each by definition the tree with the maximal contraction of a Hall' problem.

\section{Proof of Theorem 1.7}

Recall the statement of Theorem 1.7: $\eta_{\bullet}$ induces an isomorphism $\bar{\eta}: \mathcal{T}_{n} \cong H_{1}\left(\overline{\mathbb{L}}_{\bullet, n+2}\right)$. Recalling that $\mathcal{T}_{n}$ is isomorphic to $H_{0}\left(\mathbb{T}_{\bullet, n+2} ; \mathbb{Z}\right.$ ) (Proposition 2.4), we will construct a gradient vector field $\Delta$ on the chain complex $\overline{\mathbb{L}}_{\bullet, n+2}$, and then show that the resulting chain map $\phi^{\Delta} \circ$ ๓ induces an isomorphism

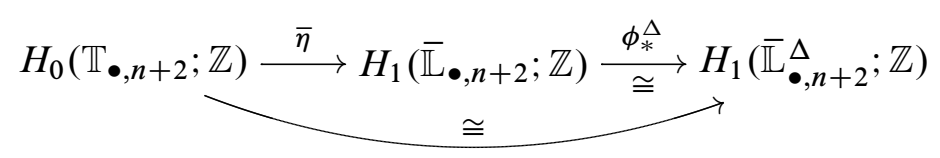

implying $\bar{\eta}$ is an isomorphism as desired (where $\phi_{*}^{\Delta}$ is an isomorphism by Theorem 3.3.) We remark that in what follows, we will think of $\overline{\mathbb{L}}_{\bullet, n+2}$ as a subset of $\mathbb{L}_{\bullet, n+2}$ rather than a quotient, so that it has a generating set of trees.

To define $\Delta$ we begin by noting that via the operation of "removing the rooted edge" each tree $J \in \overline{\mathbb{L}}_{\bullet, n+2}$ defines a unique tree $t \in \mathbb{T}_{\bullet, n+2}$ which we call the underlying tree of $J$. Specifically, if the root of $J$ is adjacent to an internal vertex of valence greater than 3 , then $t$ is gotten by deleting the root and (the interior of) its edge but leaving the internal vertex. If the root of $J$ is adjacent to a trivalent vertex, then $t$ is gotten by deleting the root and (the interior of) its edge but converting the resulting $2-$ valent vertex into a nonvertex point of $t$. This operation in the case where the root 
is adjacent to a trivalent vertex is succinctly described using the notion of the inner product $\left\langle J_{1}, J_{2}\right\rangle$ of two rooted trees $J_{1}$ and $J_{2}$, which is the unrooted tree defined by identifying the roots of $J_{1}$ and $J_{2}$ to a single nonvertex point. (The orientation of $\left\langle J_{1}, J_{2}\right\rangle$ is given by numbering the middle edge first, followed by the edges of $J_{1}$ and then the edges of $J_{2}$ in the orderings prescribed by their orientations.) Then $\left\langle J_{1}, J_{2}\right\rangle$ is the underlying tree of the bracket $\left(J_{1}, J_{2}\right)$.

Next choose a fixed basepoint for every isomorphism class of tree $t \in \mathbb{T}_{\bullet, n+2}$ in the following way. If an internal vertex is fixed by the symmetry group of $t$, choose the basepoint to be such a vertex. Otherwise, by the following lemma, the tree is of the form $t=\langle T, T\rangle$ for some tree $T$. In this case choose the basepoint of $t$ to be the midpoint of the middle edge joining the two copies of $T$.

Lemma 5.1 Every $t \in \mathbb{T}_{\bullet, n+2}$ is either of the form $\langle T, T\rangle$ or it has an internal vertex fixed by its symmetry group.

Proof The barycenter of a tree $t$ is defined as the midpoint of a maximal geodesic, and is uniquely defined. Note that the symmetry group of the tree fixes this barycenter. If the barycenter is in the middle of an edge, and one endpoint of that edge is not fixed by the symmetry group of the tree, then the tree is of the form $t=\langle T, T\rangle$ for some tree $T$.

Now define $\Delta$ as follows: If the root of $J$ is adjacent to an internal vertex of valence greater than 3 , then $\Delta(J)=0$. If $J=(T, T)$ then we also define $\Delta(J)=0$. Otherwise, $\Delta(J)$ is defined by "sliding" the rooted edge of $J$ away from the basepoint of the underlying tree until it attaches to the next internal vertex:
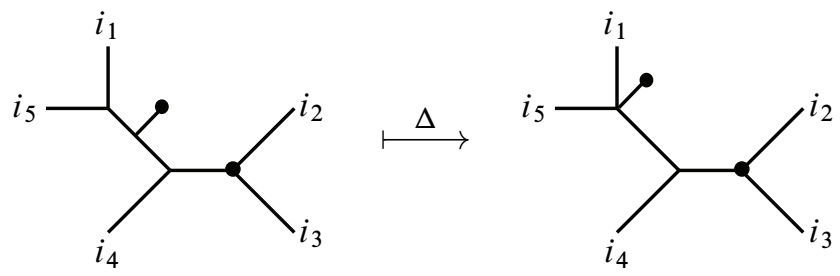

The orientation of $\Delta(J)$ is defined as follows: reorder the edges of $J$ by an even permutation so that the contracted edge is numbered 1. Then the edges of $\Delta(J)$ are ordered consistently with their order in $J$.

Lemma $5.2 \Delta: \overline{\mathbb{L}}_{\bullet, n+2} \rightarrow \overline{\mathbb{L}}_{\bullet+1, n+2}$ is a well-defined gradient vector field. 
Proof Note that $\Delta$ is well-defined as a map: Since there are no trees of the form $(i, T)$ in $\overline{\mathbb{L}}_{\bullet, n+2}$, there will always be a neighboring internal vertex to slide to as required. Also $\partial(\Delta(J))=J$ plus other terms not equal to $J$ since other expansions of $\Delta(J)$ where the root is adjacent to a trivalent vertex have the root further away from the basepoint.

We must check that $\Delta$ does not map any $\mathbb{Z}$-trees to $\mathbb{Z}_{2}$-trees, and does not map any $\mathbb{Z}_{2}$-trees to $\mathbb{Z}$-trees. We only need to check the cases where $J=\left(J_{1}, J_{2}\right)$ has the root adjacent to a trivalent vertex. Note that every symmetry of $J$ obviously fixes the root. If $J$ has a symmetry which flips the two outgoing trees $J_{1}$ and $J_{2}$, then $J_{1}=J_{2}$ and $\Delta(J)$ was defined to be 0 . If $J_{1} \neq J_{2}$, then any symmetry of $J$ restricts to the identity on the edge of the underlying tree to which the root edge attaches, so when we slide the root along this edge to get $\Delta(J)$, the symmetry is still there, and is still orientation-reversing, since it involves exchanging the same subtrees. If there are no nontrivial symmetries of $J$, then sliding the root edge along an edge $e$ to the neighboring internal vertex $v$ can not create a symmetry, as we now argue. If there were a symmetry of the contracted tree, it could not fix the edge $e$, since such a symmetry would also be present in the original tree. Thus any new symmetry would move the edge $e$ to a new edge emanating from $v$, and therefore move the entire tree $J_{e}$ growing out of $v$ which contains $e$. However, the basepoint of the underlying tree is in $J_{e}$, so that we have a symmetry that moves the basepoint, contradicting our choice of basepoints.

Let us analyze what a gradient path is in this vector field. A nonvanishing application of $\Delta$ pushes the root away from the basepoint. Now the only terms of $\partial(\Delta(J))-J$ on which $\Delta$ will evaluate nontrivially are those where the root has been pushed by $\partial$ onto another edge of the underlying tree that is further away from the basepoint. Repeated applications of $\Delta$ moves the rooted edges in such terms further and further away, until they eventually reach univalent edges, where the trees are zero in $\overline{\mathbb{L}}_{\bullet, n+2}$. Thus there are no closed gradient paths and $\Delta$ is a gradient field.

Next we analyze the critical generators. Given a tree $t \in \mathbb{T}_{\bullet, n+2}$, let $t^{b}$ denote the tree with a rooted edge attached to the basepoint. Also let $\langle T, T\rangle^{S}$ denote the tree where a rooted edge is attached to one of the endpoints of the central edge of a symmetric tree $\langle T, T\rangle$.

Lemma 5.3 The Morse complex is generated by the following two types of trees:

(1) trees of the form $t^{b}$, for $t \in \mathbb{T}_{\bullet, n+2}$

(2) trees of the form $\langle T, T\rangle^{s}$. 
Proof If $J$ is a tree not of the form $(T, T)$ and the root edge of $J$ is adjacent to a trivalent vertex, then $\Delta$ is nonzero, so $J$ is not critical. If the root attaches to a higher-valence vertex $v$ away from the basepoint of the underlying tree $t$, then consider the tree $J^{\prime}$ where the root edge attaches to the middle of the edge of $t$ adjacent to $v$ that is closer to the basepoint. Then, in most cases, $\Delta\left(J^{\prime}\right)=J$. The one case that is ruled out is the case when $J^{\prime}=(T, T)$, where $\Delta$ was defined as 0 . This explains why the tree $\langle T, T\rangle^{S}$ is critical. Finally, if the root edge attaches to the basepoint, $t$ is not in the image of $\Delta$, so it is critical.

Recall now the chain map $\eta_{\bullet}: \mathbb{T}_{\bullet} \rightarrow \overline{\mathbb{L}}_{\bullet+1}$ from Lemma 2.5 defined by summing over attaching a root to all internal vertices. We calculate $\phi^{\Delta} \circ \eta_{\bullet}$ as follows. If $t$ is not of the form $\langle T, T\rangle$, then $\phi^{\Delta}(\mathfrak{m}(t))=t^{b} \in \overline{\mathbb{L}}_{\bullet, n+2}^{\Delta}$. This is because $\Delta$ vanishes on the tree summands of $\sqcap(t)$, so $\phi^{\Delta}(\sqcap(t))$ is the sum of critical generators in $\emptyset(t)$. On the other hand, suppose $t=\langle T, T\rangle$ where $T$ is of even degree. Then $\phi^{\Delta}(\mathfrak{\curvearrowleft}(\langle T, T\rangle))=2\langle T, T\rangle^{s}$. If $T$ has odd degree, then $\emptyset(\langle T, T\rangle)=0$, because there is an orientation reversing automorphism exchanging the two endpoints of the central edge.

Now consider the chain map $\phi^{\Delta} \circ$ ๆ which will have both a kernel Ker• and a cokernel Cok• :

$$
0 \rightarrow \mathrm{Ker}_{\bullet} \rightarrow \mathbb{T}_{\bullet} \rightarrow \overline{\mathbb{L}}_{\bullet+1}^{\Delta} \rightarrow \mathrm{Cok}_{\bullet} \rightarrow 0
$$

Note that we have indexed Cok• to match $\mathbb{T}_{\bullet}$ and not $\overline{\mathbb{L}}_{\bullet+1}^{\Delta}$. Let us now analyze the kernel and cokernel. First we set up some convenient notation. A tree denoted by $A$ must have an orientation reversing automorphism. A tree denoted by $K$ must have no orientation reversing automorphism. A tree denoted by $J$ may or may not have one.

Lemma 5.4 The cokernel can be written as follows.

$$
\begin{aligned}
& \operatorname{Cok}_{4 i+2}=\mathbb{Z}\left\{\langle K, K\rangle^{s} \mid \operatorname{deg}(K)=2 i+1\right\} \oplus \mathbb{Z}_{2}\left\{\langle A, A\rangle^{s} \mid \operatorname{deg}(A)=2 i+1\right\} \\
& \operatorname{Cok}_{4 i+1}=\mathbb{Z}\{(K, K) \mid \operatorname{deg}(K)=2 i+1\} \oplus \mathbb{Z}_{2}\{(A, A) \mid \operatorname{deg}(A)=2 i+1\} \\
& \operatorname{Cok}_{4 i}=\mathbb{Z}_{2}\left\{\langle J, J\rangle^{s} \mid \operatorname{deg}(J)=2 i\right\} \\
& \operatorname{Cok}_{4 i-1}=\mathbb{Z}_{2}\{(J, J) \mid \operatorname{deg}(J)=2 i\}
\end{aligned}
$$

Moreover Cok• is an acyclic complex.

Proof The critical trees that are not hit by $\phi^{\Delta}{ }_{\eta_{\bullet}}$ are of two kinds: $(T, T)$ and $\langle T, T\rangle^{s}$. No multiple of $(T, T)$ is in the image, whereas $2\langle T, T\rangle^{S}$ is in the image if and only if $T$ has even degree.

If $T$ is of odd degree and itself has an orientation-reversing automorphism, then both $(T, T)$ and $\langle T, T\rangle^{S}$ will be 2-torsion, accounting for the $\mathbb{Z}_{2}$-summands in degrees 
$4 i+1$ and $4 i+2$ above. If $T$ has no such automorphism, then neither does $\langle T, T\rangle^{S}$ nor $(T, T)$, accounting for the $\mathbb{Z}$-summands. Finally, when $T$ has even degree, $2\langle T, T\rangle^{S}$ is in the image of $\phi^{\Delta}{ }_{\eta}$. and $(T, T)$ is 2 -torsion, accounting for the remaining terms above.

Notice that $\partial^{\Delta}\langle J, J\rangle^{S}=(J, J)$, because one term of $\partial$ is critical and equal to $(J, J)$ and the other terms lead to gradient flows that push the root away from the basepoint and which eventually terminate in 0 . Thus $\mathrm{Cok}_{4 i} \rightarrow \mathrm{Cok}_{4 i-1}$ is a direct sum of acyclic complexes of the form $\mathbb{Z}_{2} \rightarrow \mathbb{Z}_{2}$. Similarly $\operatorname{Cok}_{4 i+2} \rightarrow \operatorname{Cok}_{4 i+1}$ is a direct sum of acyclic complexes either of the form $\mathbb{Z}_{2} \rightarrow \mathbb{Z}_{2}$ or $\mathbb{Z} \rightarrow \mathbb{Z}$. Therefore Cok• is acyclic.

Now we turn to an analysis of Ker॰. Again a tree denoted $A$ must have an orientation reversing automorphism.

Lemma 5.5 The kernel can be written as follows.

$$
\begin{aligned}
& \operatorname{Ker}_{4 i+2}=\mathbb{Z}_{2}\{\langle J, J\rangle \mid \operatorname{deg}(J)=2 i-1\} \\
& \operatorname{Ker}_{4 i+1}=0 \\
& \operatorname{Ker}_{4 i}=\mathbb{Z}_{2}\{\langle A, A\rangle \mid \operatorname{deg}(A)=2 i\} \\
& \operatorname{Ker}_{4 i-1}=0
\end{aligned}
$$

Proof Clearly $\phi^{\Delta} \circ \eta_{\bullet}$ is injective away from symmetric trees $\langle T, T\rangle$. If the degree of $T$ is odd, these are all in the kernel. If the degree of $T$ is even, then $\langle T, T\rangle \mapsto$ $2\langle T, T\rangle^{S}$, and so is nonzero unless $\langle T, T\rangle$ is 2 -torsion, implying that $T$ has an orientation-reversing automorphism.

We are interested in establishing that $\eta_{0}$ induces an isomorphism on homology. This will follow because $\mathrm{Ker}_{0}$ is generated by trees which are zero in $\mathcal{T}_{n}$ : in degree $0, A$ will contain a subtree of the form $(J, J)$. Apply IHX to the base edge of this copy of $(J, J)$ to see that $\langle A, A\rangle=0$ in $\mathcal{T}_{n}$.

Formally, we argue by splitting the exact sequence into two short exact sequences:

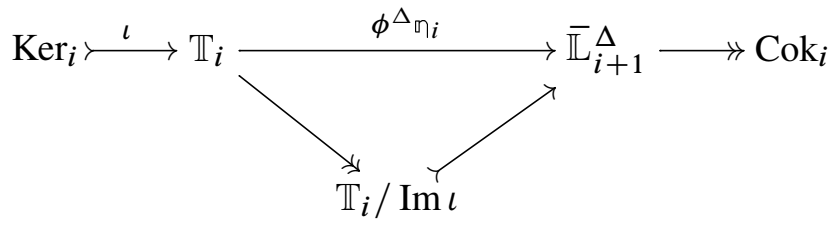


Then because the inclusion $\iota_{0}: H_{0}\left(\mathrm{Ker}_{\bullet}\right) \rightarrow H_{0}\left(\mathbb{T}_{\bullet}\right)$ induces the zero map, we have that $H_{0}\left(\mathbb{T}_{\bullet}\right) \cong H_{0}\left(\mathbb{T}_{\bullet} / \operatorname{Im} \iota\right)$. On the other hand, the cokernel is acyclic, so that $H_{i}\left(\mathbb{T}_{\bullet} / \operatorname{Im} \iota\right) \cong H_{i+1}\left(\overline{\mathbb{L}}_{\bullet}\right)$ for all $i$. Therefore

$$
\mathcal{T}_{n} \cong H_{0}\left(\mathbb{T}_{\bullet}\right) \cong H_{1}\left(\overline{\mathbb{L}}_{\bullet}^{\Delta}\right) \cong H_{1}\left(\overline{\mathbb{L}}_{\bullet}\right) \cong \mathrm{D}_{n}^{\prime} .
$$

This completes the proof of Theorem 1.7.

Remark It follows from the above discussion that $\eta_{\bullet}$ does not induce an isomorphism $H_{i}\left(\mathbb{T}_{\bullet}\right) \rightarrow H_{i+1}\left(\overline{\mathbb{L}}_{\bullet}\right)$ for arbitrary $i$. Indeed, at the next degree there is an exact sequence

$$
0 \rightarrow H_{1}\left(\mathbb{T}_{\bullet}\right) \rightarrow H_{2}\left(\overline{\mathbb{L}}_{\bullet}\right) \rightarrow \mathbb{Z}_{2}\{\langle A, A\rangle \mid \operatorname{deg}(A)=0\} \rightarrow 0 .
$$

This demonstrates a failure of surjectivity of $\eta_{1}$. In general $\eta$ can also fail to be injective. For example, $\langle(1,1,2),(1,1,2)\rangle \in \mathbb{T}_{2}$ represents a nontrivial 2-torsion homology class, which can be easily checked since there is only one degree 3 tree with the same signature. This tree is clearly in the kernel of $\eta_{2}$.

\section{Comparing filtrations of the group of homology cylinders}

Let $\Sigma_{g, 1}$ denote the compact oriented surface of genus $g$ with one boundary component. Following Garoufalidis and Levine $[11 ; 22]$, we define a homology cylinder over $\Sigma_{g, 1}$ to be a compact oriented 3-manifold $M$ equipped with a homeomorphism $m: \partial\left(\Sigma_{g, 1} \times\right.$ $[-1,1]) \cong \partial M$ such that the two maps $m_{ \pm}=\left.m\right|_{\Sigma_{g, 1} \times \pm 1}$, when composed with the inclusion $\partial M \subset M$, give an isomorphism on homology. This definition differs slightly from Habiro and Massuyeau [18], who reserve the term "homology cylinder" for those homology cylinders with a trivial action on $H_{*}\left(\Sigma_{g, 1}\right)$. Conveniently, the two definitions coincide for positive order in the Johnson filtration, which is all we will consider anyway. (See the remark at the end of the next paragraph.) Two homology cylinders $M_{0}$ and $M_{1}$ are said to be homology cobordant if there is a compact oriented 4-manifold $W$ with $\partial W=M_{0} \cup_{\partial}\left(-M_{1}\right)$, such that the inclusions $M_{i} \hookrightarrow W$ are homology isomorphisms. This defines an equivalence relation on the set of homology cylinders. Let $\mathcal{H}_{g}$ be the set of homology cylinders up to homology cobordism over $\Sigma_{g, 1}$. $\mathcal{H}_{g}$ is a group via the "stacking" operation.

Adapting the usual string link definition, Garoufalidis and Levine [11] introduced an Artin-type representation $\sigma_{n}: \mathcal{H}_{g} \rightarrow A_{0}\left(F / F_{n+1}\right)$ where $F$ is the free group on $2 g$ generators, and $A_{0}\left(F / F_{n+1}\right)$ is the group of automorphisms $\phi$ of $F / F_{n+1}$ such that there is a lift $\widetilde{\phi}: F \rightarrow F$ which is an endomorphism that fixes the product $\left[x_{1}, y_{1}\right] \cdots\left[x_{g}, y_{g}\right]$ modulo $F_{n+2}$. Here $\left\{x_{i}, y_{i}\right\}_{i=1}^{g}$ is a standard symplectic basis 
for $\Sigma_{g, 1}$. The Johnson (relative weight) filtration of $\mathcal{H}_{g}$ is defined by $\mathbb{J}_{n}=\operatorname{Ker} \sigma_{n}$. Define the associated graded group $J_{n}=\mathbb{J}_{n} / \mathbb{J}_{n+1}$. Garoufalidis and Levine show that $\mathrm{J}_{n} \cong \mathrm{D}_{n}$. (We remark that the subgroup $\mathbb{J}_{1}<\mathcal{H}_{g}$ is spanned by what Habiro and Massuyeau call homology cylinders.)

On the other hand, there is a filtration related to Goussarov-Habiro's theory of finite type 3-manifold invariants. We define the relation of $n$-equivalence (also known as $A_{n}$ - or $Y_{n}$-equivalence) to be generated by the following move: $M \sim_{n} M^{\prime}$ if $M^{\prime}$ is diffeomorphic to $M_{C}$, for some connected graph clasper $C$ with $n$ nodes. Let $\mathbb{Y}_{n}$ be the subgroup of $\mathcal{H}_{g}$ of all homology cylinders $n$-equivalent to the trivial one, and let $\mathrm{Y}_{n}=\mathbb{Y}_{n} / \sim_{n+1}$.

As Levine observes, $\mathbb{Y}_{n} \subset \mathbb{J}_{n}$, giving rise to a map of associated graded groups $Y_{n} \rightarrow J_{n}$. Rationally, Levine showed that this map is an isomorphism, and in fact both $Y_{n}$ and $J_{n}$ are rationally isomorphic to $\mathcal{T}_{n}=\mathcal{T}_{n}(2 g)$. This is implied by the following theorem, proven in the pair of papers [22;23], and stated in the introduction of [23]. It uses a surjective clasper surgery map $\theta_{n}: \mathcal{T}_{n} \rightarrow \mathrm{Y}_{n}$ introduced by Habiro [16], and further elucidated in [18, Remarks 6.6 and 7.8].

Theorem 6.1 (Levine) For $n>1$, there is a commutative diagram

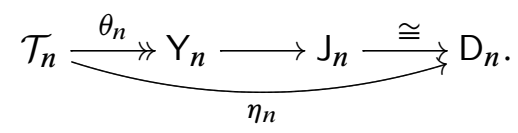

Moreover, for all $n>1, \eta_{n}$, and hence all of the maps, are rational isomorphisms.

Habiro showed that for $n=1$ we have the exact sequence $0 \rightarrow\left(\mathbb{Z}_{2} \otimes L_{2}^{\prime}\right) \oplus \mathbb{Z}_{2} \rightarrow Y_{1} \rightarrow$ $J_{1} \rightarrow 0$, although the expression of the kernel as $\left(\mathbb{Z}_{2} \otimes L_{2}^{\prime}\right) \oplus \mathbb{Z}_{2}$ is not canonical. The story for $n>1$ is more subtle over the integers, and Levine conjectured the statements in the following theorem, which are straightforward consequences of the fact that $\eta^{\prime}$ is an isomorphism.

Theorem 6.2 There are exact sequences

$$
\begin{array}{ll}
0 \rightarrow \mathrm{Y}_{2 n} \rightarrow \mathrm{J}_{2 n} \rightarrow \mathbb{Z}_{2} \otimes \mathrm{L}_{n+1} \rightarrow 0 & (n \geq 1), \\
\mathbb{Z}_{2}^{m} \otimes \mathrm{L}_{n} \rightarrow \mathrm{Y}_{2 n-1} \rightarrow \mathrm{J}_{2 n-1} \rightarrow 0 & (n \geq 2) .
\end{array}
$$

Levine did not conjecture that the map $\mathbb{Z}_{2}^{m} \otimes L_{n} \rightarrow Y_{2 n-1}$ is injective, and in fact it is not injective, basically because the framing relations discussed in [5] are also present in this context. As we prove in [3], for odd numbers of the form $4 n-1$ this allows us to get a sharp answer to what the kernel of $\mathrm{Y}_{4 n-1} \rightarrow J_{4 n-1}$ is, while for odd numbers 
of the form $4 n+1$ we determine it up to a possible quotient $\mathbb{Z}_{2} \otimes \mathrm{L}_{n+1}$. Let $\mathrm{K}_{2 k+1}^{\mathrm{Y}}$ be the kernel of the natural epimorphism $\mathrm{Y}_{2 k+1} \rightarrow J_{2 k+1}$.

Theorem 6.3 [3] For $n \geq 1$, there are exact sequences

(1) $0 \rightarrow \mathbb{Z}_{2} \otimes \mathrm{L}_{2 n+1} \rightarrow \mathrm{Y}_{4 n-1} \rightarrow \mathrm{J}_{4 n-1} \rightarrow 0$,

(2) $\mathbb{Z}_{2} \otimes \mathrm{L}_{n+1} \stackrel{a_{n+1}}{\rightarrow} \mathrm{K}_{4 n+1}^{\mathrm{Y}} \rightarrow \mathbb{Z}_{2} \otimes \mathrm{L}_{2 n+2} \rightarrow 0$.

Brief sketch of proof of (1) In [3] we prove the following two facts:

(a) In [3, Proposition 3.1] we show that Habiro's surgery map $\theta_{2 n-1}: \mathcal{T}_{2 n-1} \rightarrow$ $\mathrm{Y}_{2 n-1}$ induces an epimorphism $\tilde{\theta}_{2 n-1}: \widetilde{\mathcal{T}}_{2 n-1} \rightarrow \mathrm{Y}_{2 n-1}$ for $n \geq 2$, where $\tilde{\mathcal{T}}_{2 n-1}$ is the quotient of $\mathcal{T}_{2 n-1}$ by framing relations. (See [5] for the full definition of $\tilde{\mathcal{T}}_{2 n-1 .}$.)

(b) In [3, Proposition 3.2] we construct an epimorphism $\kappa_{2 n-1}: \mathrm{K}_{2 n-1}^{\mathrm{Y}} \rightarrow \mathbb{Z}_{2} \otimes \mathrm{L}_{n+1}$.

If follows that there is a commutative diagram of short exact sequences:

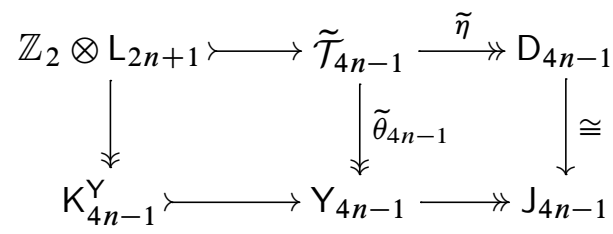

The exactness of the top row follows from the Levine conjecture and is established by combining Theorems 5.1 and 6.5(ii) of [5]. The right hand vertical arrow is the inverse of the Johnson homomorphism, which is an isomorphism, and the right-hand square is commutative by Theorem 6.1. Thus there is an induced epimorphism $\mathbb{Z}_{2} \otimes \mathrm{L}_{2 n+1} \rightarrow \mathrm{K}_{4 n-1}^{\mathrm{Y}}$ which makes the diagram commute. Considering the dimensions of domain and range as $\mathbb{Z}_{2}$-vector spaces, this left hand vertical arrow is an isomorphism by (b).

The calculation of the kernel $\mathrm{K}_{4 n+1}^{\mathrm{Y}}$ is thus reduced to the calculation of $\operatorname{Ker}\left(a_{n+1}\right)$. This is the precise analog of the question "how nontrivial are the higher-order Arf invariants?" in the setting of Whitney tower filtrations of classical links (compare the $a_{n+1}$ in Theorem 6.3 with the maps $\alpha_{n+1}$ defined in $\left.[6 ; 5 ; 4]\right)$.

Conjecture 6.4 The homomorphisms $a_{n+1}$ are injective for all $n \geq 1$, implying that there is an exact sequence $0 \rightarrow \mathbb{Z}_{2} \otimes \mathrm{L}_{2 n+2}^{\prime} \rightarrow \mathrm{Y}_{4 n+1} \rightarrow \mathrm{J}_{4 n+1} \rightarrow 0$.

Notice that, combined with Theorem 6.3(1), this conjecture implies that there is an exact sequence $0 \rightarrow \mathbb{Z}_{2} \otimes \mathrm{L}_{k+2}^{\prime} \rightarrow \mathrm{Y}_{2 k+1} \rightarrow \mathrm{J}_{2 k+1} \rightarrow 0$ for all $k \geq 1$.

To prove Theorem 6.2 we will use the following proposition proven by Levine in [23; 24], and the commutative diagram of Theorem 6.1. 
Proposition 6.5 (Levine) The following sequences are exact.

$$
\begin{gathered}
0 \rightarrow \mathbb{Z}_{2}^{m} \otimes \mathrm{L}_{n} \rightarrow \mathrm{D}_{2 n-1}^{\prime} \rightarrow \mathrm{D}_{2 n-1} \rightarrow 0 \\
0 \rightarrow \mathrm{D}_{2 n}^{\prime} \rightarrow \mathrm{D}_{2 n} \rightarrow \mathbb{Z}_{2} \otimes \mathrm{L}_{n+1} \rightarrow 0
\end{gathered}
$$

Proof of Theorem 6.2 In the even case, we get the diagram

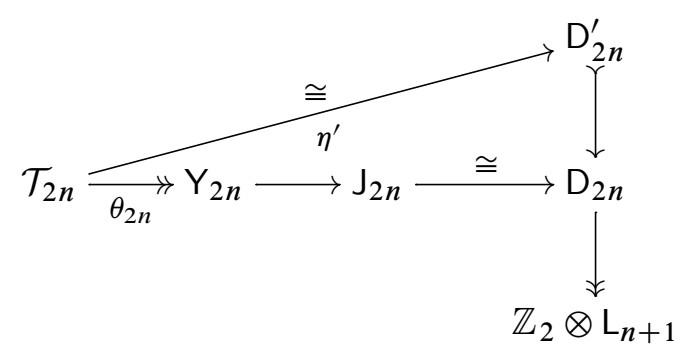

from which it follows that the map $Y_{2 n} \rightarrow J_{2 n}$ is injective with cokernel $\mathbb{Z}_{2} \otimes L_{n+1}$. Indeed after making various identifications using the isomorphisms in the diagram, we get a commutative diagram

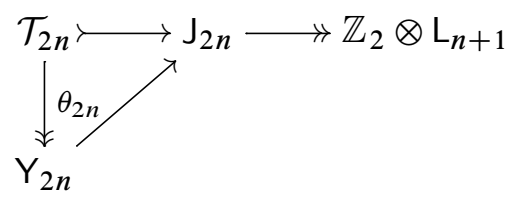

where the top row is exact. By commutativity of the triangle, $\theta_{2 n}$ is an isomorphism. Turning to the odd case, we have a commutative diagram

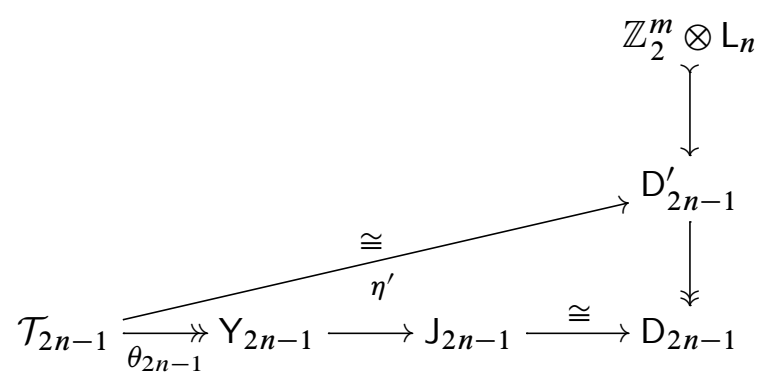

which collapses, after identifications, to the following diagram, where the top row is exact.

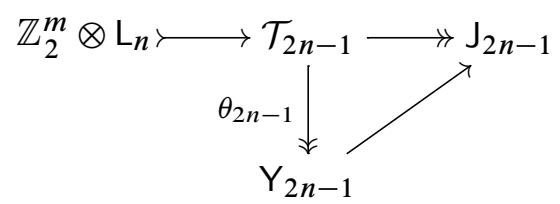

Thus the map $Y_{2 n-1} \rightarrow J_{2 n-1}$ is surjective, and $\mathbb{Z}_{2}^{m} \otimes L_{n}$ maps onto the kernel. 


\section{References}

[1] D Cheptea, K Habiro, G Massuyeau, A functorial LMO invariant for Lagrangian cobordisms, Geom. Topol. 12 (2008) 1091-1170 MR2403806

[2] T D Cochran, Derivatives of links: Milnor's concordance invariants and Massey's products, Mem. Amer. Math. Soc. 84, no. 427, Amer. Math. Soc. (1990) MR1042041

[3] J Conant, R Schneiderman, P Teichner, Geometric filtrations of string links and homology cylinders arXiv:1202.2482

[4] J Conant, R Schneiderman, P Teichner, Milnor invariants and twisted Whitney towers arXiv:1102.0758

[5] J Conant, R Schneiderman, P Teichner, Whitney tower concordance of classical links arXiv:1202.3463

[6] J Conant, R Schneiderman, $\mathbf{P}$ Teichner, Higher-order intersections in lowdimensional topology, Proc. Natl. Acad. Sci. USA 108 (2011) 8131-8138 MR2806650

[7] J Conant, K Vogtmann, On a theorem of Kontsevich, Algebr. Geom. Topol. 3 (2003) 1167-1224 MR2026331

[8] J Conant, K Vogtmann, Morita classes in the homology of automorphism groups of free groups, Geom. Topol. 8 (2004) 1471-1499 MR2119302

[9] R Forman, Morse theory for cell complexes, Adv. Math. 134 (1998) 90-145 MR1612391

[10] S Garoufalidis, M Goussarov, M Polyak, Calculus of clovers and finite type invariants of 3-manifolds, Geom. Topol. 5 (2001) 75-108 MR1812435

[11] S Garoufalidis, J Levine, Tree-level invariants of three-manifolds, Massey products and the Johnson homomorphism, from: "Graphs and patterns in mathematics and theoretical physics", (M Lyubich, L Takhtajan, editors), Proc. Sympos. Pure Math. 73, Amer. Math. Soc. (2005) 173-203 MR2131016

[12] M Goussarov, Finite type invariants and n-equivalence of 3-manifolds, C. R. Acad. Sci. Paris Sér. I Math. 329 (1999) 517-522 MR1715131

[13] N Habegger, Milnor, Johnson and tree-level perturbative invariants, preprint (2000) Available at http://www.math.sciences.univ-nantes.fr/ habegger/ PS/john100300.ps

[14] N Habegger, G Masbaum, The Kontsevich integral and Milnor's invariants, Topology 39 (2000) 1253-1289 MR1783857

[15] N Habegger, W Pitsch, Tree level Lie algebra structures of perturbative invariants, J. Knot Theory Ramifications 12 (2003) 333-345 MR1983089

[16] K Habiro, Claspers and finite type invariants of links, Geom. Topol. 4 (2000) 1-83 MR1735632 
[17] K Habiro, G Massuyeau, Symplectic Jacobi diagrams and the Lie algebra of homology cylinders, J. Topol. 2 (2009) 527-569 MR2546585

[18] K Habiro, G Massuyeau, From mapping class groups to monoids of homology cobordisms: a survey, preprint (2010) to appear in "Handbook of Teichmüller theory, Vol. III" (A Papadopoulos, editor)

[19] D Johnson, A survey of the Torelli group, from: "Low-dimensional topology (San Francisco, Calif., 1981)", (S J Lomonaco, Jr, editor), Contemp. Math. 20, Amer. Math. Soc. (1983) 165-179 MR718141

[20] M Kontsevich, Formal (non)commutative symplectic geometry, from: “The Gel'fand Mathematical Seminars, 1990-1992”, (L Corwin, I Gel'fand, J Lepowsky, editors), Birkhäuser, Boston (1993) 173-187 MR1247289

[21] D N Kozlov, Discrete Morse theory for free chain complexes, C. R. Math. Acad. Sci. Paris 340 (2005) 867-872 MR2151775

[22] J Levine, Homology cylinders: an enlargement of the mapping class group, Algebr. Geom. Topol. 1 (2001) 243-270 MR1823501

[23] J Levine, Addendum and correction to: "Homology cylinders: an enlargement of the mapping class group” [22], Algebr. Geom. Topol. 2 (2002) 1197-1204 MR1943338

[24] J Levine, Labeled binary planar trees and quasi-Lie algebras, Algebr. Geom. Topol. 6 (2006) 935-948 MR2240921

[25] W Magnus, A Karrass, D Solitar, Combinatorial group theory: Presentations of groups in terms of generators and relations, revised edition, Dover, New York (1976) MR0422434

[26] J Milnor, Link groups, Ann. of Math. 59 (1954) 177-195 MR0071020

[27] J Milnor, Isotopy of links. Algebraic geometry and topology, from: "A symposium in honor of S Lefschetz", (R H Fox, D C D C Spencer, A W Tucker, editors), Princeton Univ. Press (1957) 280-306 MR0092150

[28] S Morita, Abelian quotients of subgroups of the mapping class group of surfaces, Duke Math. J. 70 (1993) 699-726 MR1224104

[29] S Morita, Structure of the mapping class groups of surfaces: a survey and a prospect, from: "Proceedings of the Kirbyfest (Berkeley, CA, 1998)", (J Hass, M Scharlemann, editors), Geom. Topol. Monogr. 2, Geom. Topol. Publ., Coventry (1999) 349-406 MR1734418

[30] K E Orr, Homotopy invariants of links, Invent. Math. 95 (1989) 379-394 MR974908

[31] M A Readdy, The pre-WDVV ring of physics and its topology, Ramanujan J. 10 (2005) 269-281 MR2195566

[32] C Reutenauer, Free Lie algebras, London Math. Soc. Monogr. (NS) 7, Oxford Science Publ., The Clarendon Press, Oxford Univ. Press, New York (1993) MR1231799 
Department of Mathematics, University of Tennessee Knoxville TN 37996, USA

Department of Mathematics and Computer Science, Lehman College, CUNY 250 Bedford Park Boulevard West, Bronx NY 10468-1589, USA

Department of Mathematics, University of California, Berkeley and MPIM Bonn Berkeley CA 94720-3840, USA

jconant@math.utk.edu, robert.schneiderman@lehman.cuny.edu, teichner@mac.com

http://comet. lehman. cuny.edu/schneiderman/, http://math. berkeley.edu/ teichner/

Proposed: Rob Kirby

Received: 13 December 2010

Seconded: David Gabai, Cameron Gordon

Revised: 16 January 2012 